\title{
Cavity-enhanced direct frequency comb spectroscopy
}

\author{
Michael J. Thorpe and Jun Ye \\ JILA, National Institute of Standards and Technology and University of Colorado, Boulder, CO 80309-0440, USA \\ Department of Physics, University of Colorado, Boulder, CO 80309-0390, USA
}

\begin{abstract}
Cavity-enhanced direct frequency comb spectroscopy combines broad spectral bandwidth, high spectral resolution, precise frequency calibration, and ultrahigh detection sensitivity, all in one experimental platform based on an optical frequency comb interacting with a high-finesse optical cavity. Precise control of the optical frequency comb allows highly efficient, coherent coupling of individual comb components with corre-
\end{abstract}

review we will discuss the principle of cavity-enhanced direct frequency comb spectroscopy and the various imsponding resonant modes of the high-finesse cavity. The long cavity lifetime dramatically enhances the effective interaction between the light field and intracavity matter, increasing the sensitivity for measurement of optical losses by a factor that is on the order of the cavity finesse. The use of low-dispersion mirrors permits almost the entire spectral bandwidth of the frequency comb to be employed for detection, covering a range of $\sim 10 \%$ of the actual optical frequency. The light transmitted from the cavity is spectrally resolved to provide a multitude of detection channels with spectral resolutions ranging from a several gigahertz to hundreds of kilohertz. In this plementations of such systems. In particular, we discuss several types of UV, optical, and IR frequency comb sources and optical cavity designs that can be used for specific spectroscopic applications. We present several cavity-comb coupling methods to take advantage of the broad spectral bandwidth and narrow spectral components of a frequency comb. Finally, we present a series of experimental measurements on trace gas detections, human breath analysis, and characterization of cold molecular beams. These results demonstrate clearly that the wide bandwidth and ultrasensitive nature of the femtosecond enhancement cavity enables powerful real-time detection and identification of many molecular species in a massively parallel fashion. 


\section{Introduction}

Many applications ranging from scientific to commercial interests will benefit from a highly sensitive, broad bandwidth, high spectral resolution, and easy to use method for optical absorption spectroscopy. In the commercial world alone, the number of applications for optical detection of trace molecules has increased enormously in the recent years, ranging from detection of contaminants for semiconductor processing [1] and human breath analysis [2] to air quality monitoring [3] and detection of biologically hazardous or explosive materials [4].

Each of these applications has been facilitated by the development of new laser sources to access spectral domains of interest, combined with cavity enhancement techniques for increased detection sensitivity $[5]$. For example, the advent of tunable laser diodes has led to sensitive measurements of impurity levels in gases such as phosphine used in semiconductor productions 1 and for monitoring ethylene in fruit storage facilities [6]. Subsequently, quantum cascade lasers and optical parametric oscillators operating in the mid-infrared have been applied to human breath analysis for health screening and optical detection of explosives [7] 8. These innovations represent remarkable advances in our capability to monitor and understand our environment. However, these technologies still operate in relatively narrow spectral regions and thus the number of different molecules that can be studied or detected with a single system is very small. This severely limits our capability of performing surveyance on a global scale in real time.

In the scientific community, sensitive and high resolution spectroscopy over a large spectral window is useful for many areas of research. For instance, studies of atmospheric chemistry, climate changes, and pollution can benefit a great deal from the capability to monitor multiple molecules simultaneously [9] [10]. Investigations of molecular potential energy surfaces, energy level structure, local and eigen-mode dynamics, intramolecular energy redistributions, as well as interaction dynamics and chemical reactions all desire spectroscopic probes with high spectral resolution and accuracy over a wide range of spectral coverage [11] 12]. For the emerging field of ultracold molecules, our ability to photoassociate cold molecules from pairs of free atoms depends on extensive spectroscopic probes to find the most efficient photoassociation pathways [13]. To study a new class of collision and reaction dynamics in the low energy limit [14] 15], the need for higher resolution and sensitivity while under a broad spectral coverage is now of more urgent importance [16] 17].

Optical frequency combs based on mode-locked femtosecond lasers provide hundreds of thousands of sharp spectral components - frequency comb teeth - evenly distributed across hundreds of nanometers of optical spectral bandwidth. Direct frequency comb spectroscopy takes advantage of this unique property of frequency comb to recover high resolution spectral features across the entire 
manifold of atomic and molecular energy levels of interest [18 [19]20. The development of mode-locked fiber lasers has led to robust optical frequency comb sources capable of continuous operations without user intervention 21. Frequency comb-based systems can also employ high peak intensities available from the pulsed output to enable efficient nonlinear frequency conversions for easy access to spectral regions spanning from the UV to the far infrared 22, 23. While techniques such as fourier transform infrared spectroscopy (FTIR) also offer broad spectral coverage with reasonably good spectral resolution, the intrinsically parallel detection capability enabled by a frequency comb brings a truly revolutionary impact to the efficiency of signal collection. Recent approaches to molecular detection that use broad bandwidth frequency combs have indeed created parallel detection schemes that in a single shot record large spectral bandwidths [24] 25]. The only missing piece in this powerful combination for spectroscopy is high sensitivity, which can be satisfactorily addressed via the use of high-finesse, broadband, low-dispersion enhancement cavities [26] [27].

Frequency comb based spectroscopic systems have made significant progress recently [28] 29] [30]. While they are not yet as simple as an FTIR-like spectrometer, the demonstrated capability of providing cavity-enhanced sensitivity over hundreds of nanometers of optical wavelength has stimulated a great interest in the spectroscopy community. It is our hope that this review article will provide useful material to facilitate the spread of this new spectroscopic technique to the wider community. In the article we present a review of cavity-enhanced direct frequency comb spectroscopy (CE-DFCS), discussing the principles of operations followed by several examples of applications. To provide useful technical information, a number of optical frequency comb sources will be surveyed, including titanium sapphire lasers, erbium fiber lasers, yetterbium fiber lasers, and other comb sources generated through nonlinear frequency conversion. Also, we will present a number of methods for coupling a frequency comb to an optical cavity and discuss various techniques to provide parallel detections of frequencyresolved absorption information transmitted from the optical cavity. Methods for increasing the spectral bandwidth and reducing acquisition time in future systems will be discussed. Finally, a series of CE-DFCS measurements will be presented to demonstrate the current capabilities of these systems.

\section{Frequency comb properties and sources}

\subsection{Comb properties}

Many of the same properties that make the frequency comb an excellent tool for counting optical frequencies and transferring coherence across vast spectral regions also make it an ideal light source for broadband, cavity enhanced spectroscopy. Frequency combs are capable of covering very large spectral ranges of hundreds of tera- 
hertz, thus enabling investigations of global energy level structure for multiple species. At the same time, the spectrally sharp and precisely defined frequency structure of the comb components allow high-resolution and frequency-calibrated spectroscopy across massively parallel detection channels. For the same reason, the frequency comb is ideal for sensitive intracavity spectroscopy since it can be efficiently coupled to a corresponding set of resonant modes of a high finesse optical cavity across a wide spectral region.

\section{Time domain}

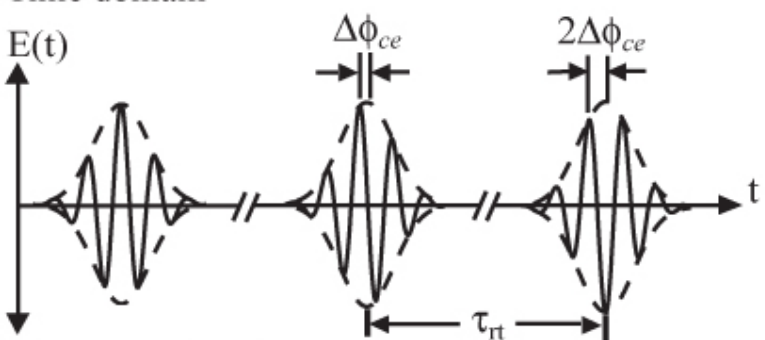

\section{Frequency domain}

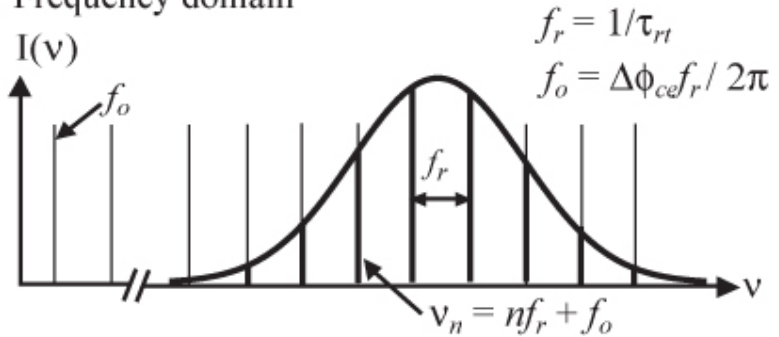

Fig. 1 The time and frequency domain properties of an optical frequency comb. The top panel shows the time domain representation of a mode-locked pulse train including the temporal spacing between pulses $\left(\tau_{r t}\right)$ and the evolution of the carrier envelope phase $\left(\Delta \phi_{c e}\right)$. The bottom panel shows the frequency domain picture where the evenly spaced comb modes with frequencies $\nu_{n}$ lie beneath a gaussian spectral envelope.
The frequency of each comb component is defined by the repetition frequency $\left(f_{r}\right)$ and the carrier-envelope offset frequency $\left(f_{o}\right)$ via the simple relation $\nu_{n}=\mathrm{n} f_{r}+$ $f_{o}$ 31. The time and frequency domain representations of a comb are illustrated in Fig. 1. For optical frequencies in the visible and near IR, the comb mode index $n$ takes on values ranging from $10^{5}$ to $10^{7}$, depending on $f_{r}$. The typical range for $f_{r}$ is $10 \mathrm{MHz}<f_{r}<10 \mathrm{GHz}$ while $f_{o}$ can take on values of $-f_{r} / 2<f_{o}<f_{r} / 2$. Precise control of these two degrees of freedom establishes the comb as an accurate frequency tool for atomic and molecular spectroscopy and also permits efficient coupling of the comb to the modes of a high finesse passive optical cavity. To implement control, one first needs to measure $f_{r}$ and $f_{o}$. $f_{r}$ can be determined directly from the pulse train incident on a fast photodiode while $f_{o}$ requires nonlinear interferometry 31] 32. Two stable radio frequency references can be used subsequently to stabilize $f_{r}$ and $f_{o}$ via servo transducers acting on the laser cavity length and the intra-cavity dispersion. A frequency comb can also be stabilized directly to a passive optical cavity by detecting the cavity response to the incident comb spectrum in the optical domain 33, as discussed in later sections of this paper.

\subsection{Comb sources and their applications to spectroscopy}

Many different types of mode-locked laser sources exist to provide coverage for a wide variety of spectral regions from blue to the mid IR. When nonlinear frequency con- 
version and spectral broadening techniques are implemented, the spectral coverage can be extended well into the VUV and the far infrared. The diversity of spectral regions that can be accessed allow frequency comb spectroscopy to be performed on a large variety of atomic and molecular systems. For the purposes of this review, we will restrict discussions to titanium sapphire lasers, erbium fiber lasers, and yetterbium fiber lasers. Nonlinear frequency conversion to other spectral regions based on these laser sources will also be briefly discussed. Figure 2 shows spectral overlaps between various frequency comb sources and relevant regions for useful atomic and molecular spectroscopy.

While the gain bandwidth of Ti:sapphire lasers cover only 700-950 nm, subsequent super-continuum generations in photonic crystal and tapered telecommunications fibers 23 34 35, and the later development of octave-spanning Ti:sapphire lasers via intracavity nonlinear phase modulation allowed spectral coverage extending from the deep blue to the near Infrared (NIR) [36] 37 38. As the first generation of precisely controlled optical frequency combs were based on Ti:sapphire lasers, it is natural that they played important roles in the first demonstrations of direct frequency comb spectroscopy [18. Frequency comb based cavity-enhanced absorption spectroscopy (CEAS) and cavity ring-down spectroscopy (CRDS) were also first performed with Ti:sapphire combs on molecular overtone transitions 26] 27]. The secondharmonic of a Ti:sapphire comb was scanned over a low-

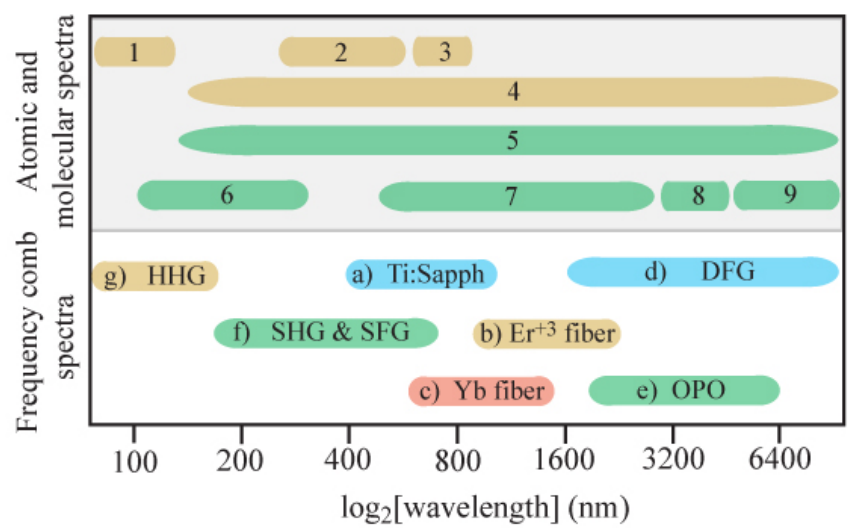

Fig. 2 Spectral coverage for several types of mode-locked lasers and corresponding atomic and molecular transitions to which they can be applied. 1, transitions from ground states of noble gas; 2 and 3, transitions from ground states of alkaline earth or alkali atoms; 4 and 5 , transitions between excited atomic or molecular states; 6 , transitions from ground states of molecules; 7 , overtones of molecular ro-vibrational transitions; 8 and 9, fundamental vibrations of light-nuclei or heavy-nuclei molecules. a) Ti:sapphire mode-locked lasers, after nonlinear spectral broadening, covers the entire visible range from 400 to $1200 \mathrm{~nm}$; b)Erbium-doped fiber lasers (centered around $1550 \mathrm{~nm}$ ) can be made to cover 1000 - 2400 $\mathrm{nm}$ via spectral broadening; c)Ytterbium-doped fiber lasers (centered around $1030 \mathrm{~nm}$ ) can cover 600 - $1600 \mathrm{~nm}$ via spectral broadening. Each of these lasers can have their spectral coverage further extended via nonlinear frequency conversion using techniques listed in (d), (e), (f), and (g). We thus have the possibility of performing comb spectroscopy for nearly all of the interested atomic and molecular transitions.

finesse cavity to measure high overtones of $\mathrm{C}_{2} \mathrm{H}_{2}$ in the blue visible region [39]. Frequency down conversion of Ti:sapphire combs has been used to produce infrared fre- 
quency combs via difference frequency generation (DFG) and optical parametric oscillation (OPO) techniques [40] 41] 42. Such infrared combs have been used to probe the strong fundamental vibration transition in molecules 43. Finally, high harmonic generation (HHG) has been used to generate frequency combs in the vacuum ultraviolet regions 44 45, and with improvement in the power [46] they could be used to probe ground state transitions of noble gases 47 .

More recently, mode-locked erbium and ytterbium fiber lasers have been developed that generate highly stable frequency combs which are pumped by inexpensive laser diodes [48] 49] [50] [51] [52]. These lasers can conveniently produce super-continuum spectra via coupling to highly nonlinear fibers. They have been frequency converted to cover the spectral domain extending from the infrared to ultra-violet, using the same techniques described for Ti:sapphire 53 [54. The fiber lasers are compact, reliable, and robust, with turn-key operations. Thus they have a very promising future for commercial applications. Recent work has used an erbium fiber comb to perform CE-DFCS on low molecular overtones for trace detection of a wide variety of molecules 28$] 30$. Finally, fiber combs are conveniently located in the 1.0 - $1.5 \mu \mathrm{m}$ spectral domain, overlapping with strong firstorder vibration overtones of many molecules. They are also ideal for frequency conversion to extend the coverage of frequency combs to the mid and far infrared, impor- tant regions to cover fundamental vibrational transitions of molecules.

\section{Useful properties for passive optical cavities}

Important properties for passive optical cavities used in CE-DFCS include the finesse for detection sensitivity, the intra-cavity dispersion for comb-cavity coupling, and the spectral bandwidth for useable spectroscopy windows. In this section we analyze these properties for a cavity constructed from two highly reflective mirrors with quarter-wave stack coatings. This analysis will illustrate why high finesse, low dispersion, and large spectral bandwidth are desirable qualities for a CE-DFCS cavity. We will discuss their intrinsic relationships and necessary compromises we need to make to achieve optimum detection. Another form of a high-finesse, low-dispersion optical cavity, i.e., a retro-reflecting prism cavity [55, will be mentioned briefly at the end of this section.

\subsection{Finesse}

The most important property of a cavity is its finesse. The finesse determines the enhancement factor for the intracavity absorption signal that can be recovered from the cavity transmission. Therefore, precise knowledge of the cavity finesse is important for making accurate measurements of the intracavity absorption. The cavity finesse $\mathcal{F}$ is proportional to the inverse of the total intracavity loss. For a cavity constructed from two mir- 
rors with negligible scattering losses, the cavity finesse is related to the mirror reflectivities $R_{1}$ and $R_{2}$ by the familiar equation $\mathcal{F}=\pi \sqrt[4]{R_{1} R_{2}} /\left(1-\sqrt{R_{1} R_{2}}\right)$ [56].

For low-loss cavities $(\mathcal{F}>1000)$, the cavity finesse is usually measured via CRDS [57]. CRDS measurements are performed by injecting laser light into a resonant mode of the cavity and then rapidly switching off the incident light. Without input, the intracavity power gradually decays at a rate that is determined by the ratio of the total cavity loss and the cavity round-trip time, i.e., the linewidth of a cavity resonance mode. The cavity decay time $\tau_{\text {cavity }}$ and finesse $\mathcal{F}$ are thus related by $\mathcal{F}=2 \pi \tau_{\text {cavity }} F S R$, where $F S R$ represents the cavity free-spectral-range frequency.

It is straightforward to use a single-frequency CW laser for CRDS measurements. For fast switching of the light source, the laser frequency can be swept quickly across the cavity resonance such that the time the laser spends on resonance with the cavity is shorter than the cavity lifetime. Another way of switching the incident light is to use an acousto-optic or electro-optic modulator that can provide switching times of less than a few hundred nanoseconds, sufficiently shorter than a typical high-finesse cavity lifetime. CRDS measurements can also be performed with an optical frequency comb if the mode spacings of the cavity and the comb are roughly matched. The frequency comb components can then be frequency-scanned across the corresponding cavity modes just as in the case for a $\mathrm{CW}$ laser. When the cavity transmitted light is frequency resolved using a grating or some other forms of dispersion, the information about the cavity finesse, and thus the intracavity loss, is obtained simultaneously across the entire spectrum of the comb 27].

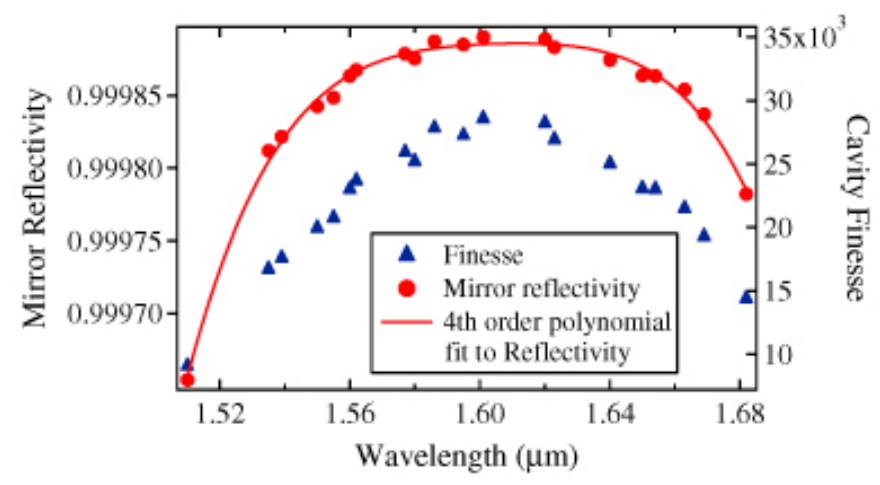

Fig. 3 The finesse, and the corresponding cavity mirror reflectivity, of an optical enhancement cavity spanning from 1.51 to $1.68 \mu \mathrm{m}$ measured by a mode-locked $\mathrm{Er}^{+3}$ fiber laser using frequency-resolved cavity ring-down spectroscopy.

A set of measurements using fast sweeping of the comb frequencies and a grating disperser after the cavity are shown in Fig. 3. Here, a frequency comb from a modelocked Er-fiber laser was used to couple into a cavity constructed from two mirrors with peak reflectivity at $1.6 \mu \mathrm{m}$ [29]. A grating was used in cavity transmission to provide a 1-nm spectral resolution. A beam splitter was used to direct a portion of the cavity transmission to an optical spectrum analyzer for wavelength calibration of the ring-down measurements. 


\subsection{Cavity mode frequencies and dispersion}

The intracavity dispersion of an optical cavity is important, especially for frequency comb-based spectroscopy, as it determines the spectral bandwidth over which the cavity modes and the comb components can be overlapped simultaneously. While the comb modes are evenly spaced in the frequency domain, $\nu_{n}=n f_{r}+f_{o}$, the cavity mode spacing is frequency dependent, $F S R=$ $c /\left(2 L+\left.c(\partial \phi / \partial \omega)\right|_{\omega}\right)$, where $L$ is the cavity length and $c$ is the speed of light. The degree to which the cavity FSR is wavelength (or optical frequency)-dependent is determined by the intracavity dispersion term $\left.c(\partial \phi / \partial \omega)\right|_{\omega}$ 58 59 60. Clearly, low intracavity dispersion is important for coupling a broad-bandwidth comb spectrum to respective cavity modes. Furthermore, precise characterizations of the intracavity dispersion are essential for the cavity filtered detection scheme described in Section 6.3.

For now we describe a method of using the frequency comb itself to precisely measure the intracavity dispersion. We will discuss the application of these measurements in later sections.

A schematic of the comb-based cavity dispersion measurement is shown in Fig. 4. The comb acts as a frequency ruler to determine the cavity mode structure and the wavelength-dependent FSR. First, $f_{r}$ of the laser is detuned away from the cavity $F S R$ at $800 \mathrm{~nm}$ as shown in Fig. 4 b). A frequency discrimination signal between the cavity modes and frequency combs is re-

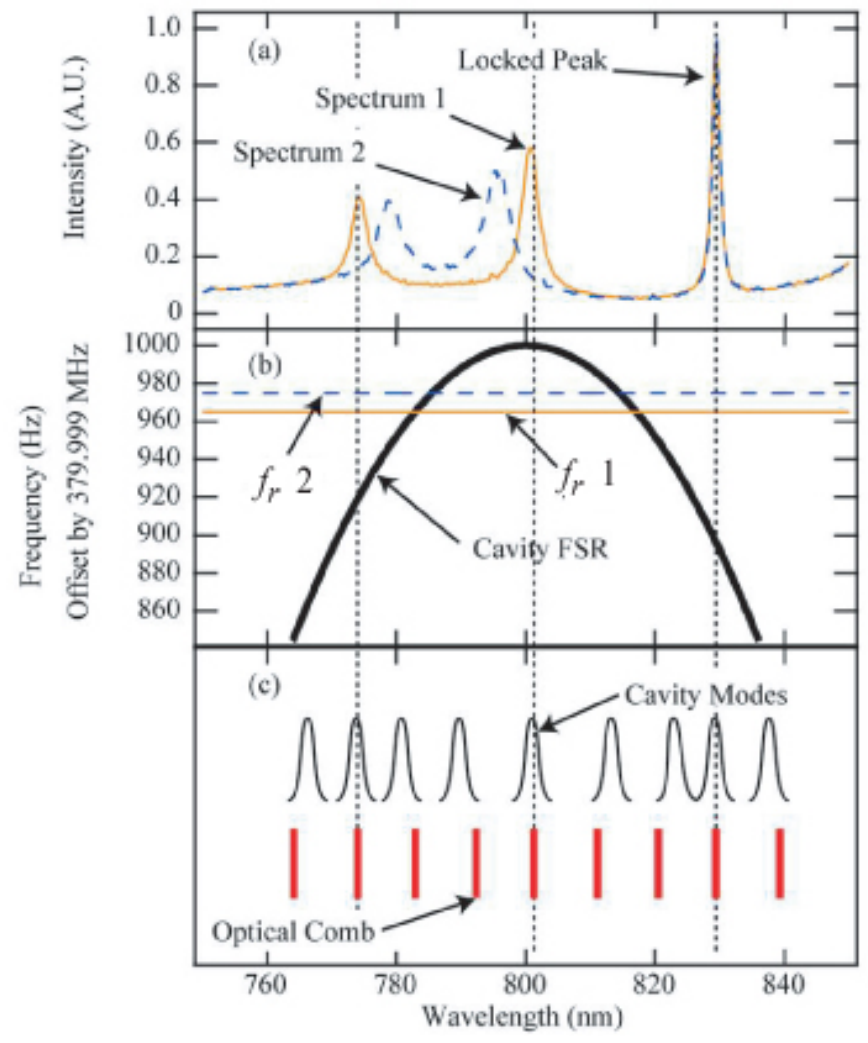

Fig. 4 A technique for using the frequency comb for measuring intracavity dispersion. (a) The cavity transmission spectrum for two values of $f_{r}$, showing the spectral regions where the comb modes and cavity modes overlap. While comb/cavity mode overlap is forced at the locking wavelength near $830 \mathrm{~nm}$, the other cavity transmission peaks arise from the frequency dependence of the cavity FSR. (b) The frequency dependent cavity $F S R$ and values of $f_{r}$ that give rise to the transmission spectra in (a). (c) A schematic of the cavity and comb modes for the case of Spectrum 1 in (a), illustrating that cavity transmission peaks occur only where there is overlap between the cavity and comb modes.

covered and serves to lock the comb to the cavity near the edge of the cavity/comb spectrum $(830 \mathrm{~nm}$ in this case). This locking wavelength becomes a reference from which the cavity $F S R$ is compared to the laser $f_{r}$. As 
one gradually moves away from $830 \mathrm{~nm}$ to other parts of the comb spectrum, the corresponding comb components will spectrally walk away from their respective cavity resonance modes. However, after a sufficiently large spectral gap, a set of comb lines will again come on resonance with cavity modes. Overlaps between the comb frequencies and cavity modes that lead to cavity transmissions are schematically illustrated in Fig. 4 c). A spectrometer is used to measure the wavelength-resolved intensity of the light transmitted from the cavity, as shown in Fig. 4

a) for two different values of $f_{r}$.

The frequency separation between the $m^{t h}$ cavity mode and the $m^{\text {th }}$ comb component from the locking point $(830 \mathrm{~nm})$ can be determined by the walk-off integral 60],

$$
\Delta \nu_{m}=\int_{\nu_{l}}^{\nu_{m}}\left(\frac{F S R(\nu)}{f_{r}\left(\nu_{m}\right)}-1\right) d \nu
$$

Here $F S R(\nu)$ is the wavelength (frequency)-dependent cavity $F S R$ that is to be determined. $\Delta \nu_{m}$ is the frequency gap between the $\mathrm{m}^{\text {th }}$ comb component and the $\mathrm{m}^{\text {th }}$ cavity mode from the locking wavelength. $\nu_{l}$ is the frequency of the locking wavelength and $\nu_{m}$ is the frequency of the $\mathrm{m}^{\text {th }}$ comb mode. When $\Delta \nu_{m}=k f_{r}(k=$ $\ldots,-2,-1,0,1,2, \ldots)$, the $m^{\text {th }}$ comb frequency overlaps with the $(m+k)^{t h}$ cavity mode. This comb component then appears as a peak in the cavity transmitted spectrum. With the laser locked to the cavity at $830 \mathrm{~nm}$, $f_{r}$ is scanned and the wavelength values of the cavity transmission peaks are recorded for each value of $f_{r}$. By differentiating the walk-off integral with respect to the comb mode number we obtain

$$
\operatorname{FSR}\left(\nu_{t}\right)=f_{r}\left(\nu_{t}\right)+\left(\nu_{t}-\nu_{l}\right) \frac{d}{d \nu_{t}} f_{r}\left(\nu_{t}\right)
$$

Here, the values of $f_{r}$ that give rise to cavity transmission peaks at frequencies $\nu_{t}$ are used to determine the frequency dependance of the cavity $F S R$. Finally, a simple equation

$$
G D D(\nu)=\frac{d}{d \nu} \frac{1}{F S R(\nu)}
$$

is used to calculate the intracavity group-delay-dispersion from the frequency dependent cavity FSR. Fig. 5 shows a measurement of the intracavity dispersion for an evacuated two-mirror cavity centered at $800 \mathrm{~nm}$ using the comb technique.

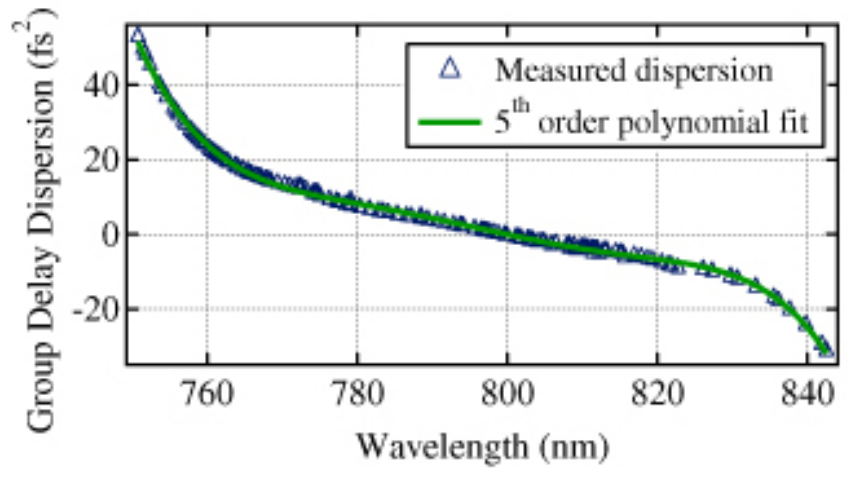

Fig. 5 Measurement of the dispersion of a two-mirror cavity with peak reflectivity at $800 \mathrm{~nm}$, using the comb-based cavity dispersion measurement technique.

The precision of this dispersion measurement technique is directly related to the cavity finesse. Higher finesse leads to more sharply peaked features in cavity 
transmission and reduced uncertainties in the determination of the center wavelength of the transmission peak. In the example for Fig. 5, the cavity under measurement had a finesse of 3000 and the achieved experimental precision for intracavity dispersion measurement was better than $1 \mathrm{fs}^{2}$.

\subsection{Spectral bandwidth}

The spectral bandwidth of a cavity refers to a spectral window in which the cavity has a high finesse and nearly uniform cavity $F S R$ that are important for cavity-enhanced frequency comb spectroscopy. The spectral bandwidth provided by quarter-wave stack Bragg reflectors depends on the refractive index contrast of the two materials that comprise the stack. Typical bandwidths are around $\sim 15 \%$ of the center optical frequency where the mirrors have their peak reflectivity. However, the spectral coverage of modern optical frequency combs can be very large ( $\sim 90 \%$ of the central frequency). As a result, optical cavity designs currently available limit the spectral coverage that can be achieved with CE-DFCS.

In response to this, efforts toward improved designs for low-loss and low-dispersion mirrors have been intense over the past few years 61. Also, new designs for highfinesse cavities that use prism retroreflectors instead of mirrors are under development. Since prism retroreflectors are based on broad bandwidth effects, such as Brewsters angle and total internal reflection, these cavities could provide spectral bandwidths of $80 \%$ of the center optical frequency with a finesse of up to 50,000 [55]. Such cavities have increased dispersion since a portion of the cavity mode is inside of the prism. However, careful design of the cavity prisms, including minimizing the prism size and the use of low dispersion materials, looks promising for providing simultaneous low dispersion and high finesse over large spectral bandwidths.

\section{Cavity/comb coupling}

In this section we discuss techniques that are used to couple the frequencies of an optical comb to the resonant modes of an optical cavity. Three techniques will be discussed, including (1) lock of the comb components to the cavity modes, (2) a low amplitude, fast sweep of the comb components over the cavity modes, and (3) precision scan between initially mismatched $F S R$ and $f_{r}$ such that one comb component leaks out of the cavity at a time. Each of these techniques offers different compromises between benefits and limitations for performing CE-DFCS. Practical applications will dictate the choice for the right approach.

\subsection{Comb locked to cavity}

The most intuitive method for coupling a frequency comb to an optical cavity is a precise and simultaneous overlap of all of the comb frequencies with corresponding cavity modes (Fig. 6). To perform this type of coupling requires 
that the optical cavity maintains the same optical path length as that of the frequency comb laser (i.e. FSR = $\left.f_{r}\right)$. Also, the dispersion of the optical cavity must be sufficiently low to ensure a large number of comb modes overlap with their corresponding cavity modes.

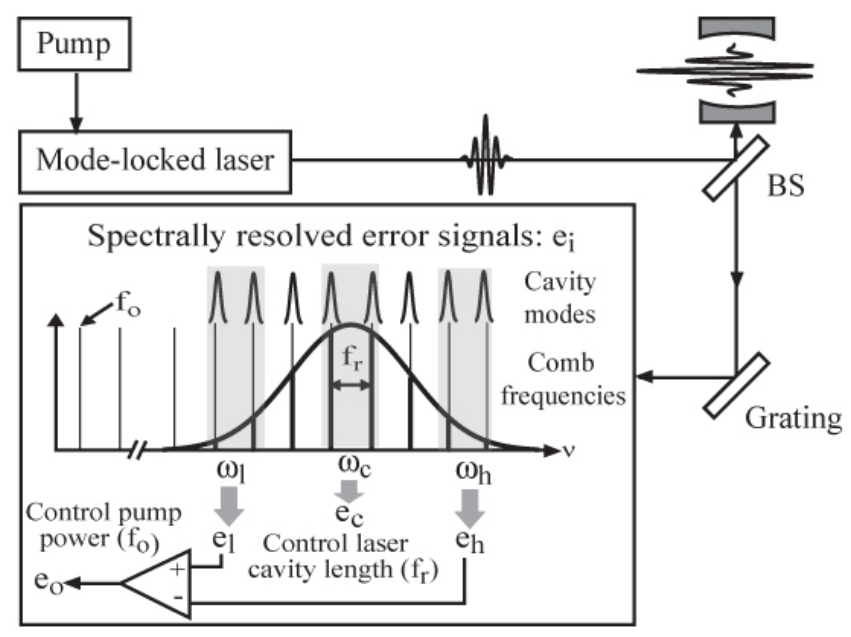

Fig. 6 A frequency comb can be locked to an optical cavity by generating spectrally resolved cavity-locking error signals.

An error signal at the center frequency is used to lock a collection of comb modes at $\omega_{c}$ to be on resonance with their respective cavity modes. Error signals at the high $\left(\omega_{h}\right)$ and low $\left(\omega_{l}\right)$ ends of the comb spectrum can be used to maintain a correct value of $f_{o}$. Adjustment of $f_{o}$ and $f_{r}$ (via the optical lock at $\omega_{c}$ ) ensures an optimal overlap of the comb and cavity modes over a wide spectral bandwidth.

For a broadband frequency comb, two servo loops are needed to lock the comb to a cavity 32 62, one for $f_{r}$ and the other for $f_{o}$. The two servo error signals are generated through spectrally resolved detection of the cavity-comb interaction as shown in Fig. 6. For robust lock, we use a modulation/lock-in detection approach.
For example, an EOM can be used to place modulation sidebands on the frequency comb, or the cavity length can be dithered rapidly. A collection of comb modes at the center of the spectrum is detected, after their interaction with their respective cavity modes. This generates an error signal that locks the optical comb frequencies to the cavity modes by acting on $f_{r}$. The interaction of the high and low frequency ends of the comb spectrum with the cavity can be used to lock $\mathrm{f}_{o}$ to the value that results in the maximum spectral bandwidth coupled into the optical cavity. These two loops act together to optimize the coupling between the comb and cavity.

The main benefit of locking the comb to the cavity is that the optical power transmitted through the cavity is nearly that of the beam incident to the cavity. Furthermore, the cavity transmission is continuously present. These two features yield a high signal to noise ratio $(S / N)$ for measurements of the cavity transmitted beam and a fast averaging time due to the continuous presence of the cavity transmission. The largest drawback is that the spectral bandwidth of the comb that is coupled into the cavity is typically limited to $<10 \%$ of the center frequency. Also, the continuous coupling of the comb to the cavity is very sensitive to acoustic and vibration-induced frequency noise that gets converted to a substantial amount of intensity noise on the cavity transmission if the servo loops do not have sufficient gains. 


\subsection{Comb swept around cavity}

The second method for coupling comb frequencies to cavity modes involves a periodic injection of the comb into the cavity. This is accomplished by introducing a low amplitude, quick frequency sweep between the corresponding modes of the cavity and the comb [26] 27]. This type of coupling has several advantages and some drawbacks compared to directly locking the comb to the cavity. The first advantage is that the requirement on the intracavity dispersion is relaxed. Since the comb and cavity modes are being swept over each other, it is no longer required that all of the comb and cavity modes are exactly overlapped simultaneously. Instead, different modes are allowed to come on resonance at slightly different times, thus increasing the useful spectral bandwidth that is recovered in cavity transmission. With a reasonably low dispersion, high finesse, and fast frequency sweeping, the time it takes for different comb modes to come on resonance with the cavity can be shorter than the cavity lifetime.

In the case of Fig. 7, $100 \mathrm{~nm}$ of comb spectrum centered at $1.55 \mu \mathrm{m}$ is swept over the cavity modes. Due to low intracavity dispersion and optimal matching of the comb to the cavity, all comb frequencies come onto resonance with the cavity at nearly the same time. Under these conditions, the cavity transmission signal is sharply peaked. The amplitude of the frequency sweep can be made very small such that the comb is on reso- nance with the cavity for a large fraction of the sweep time. By using the smallest possible sweep amplitude, the power contained in the cavity transmission is maximized.

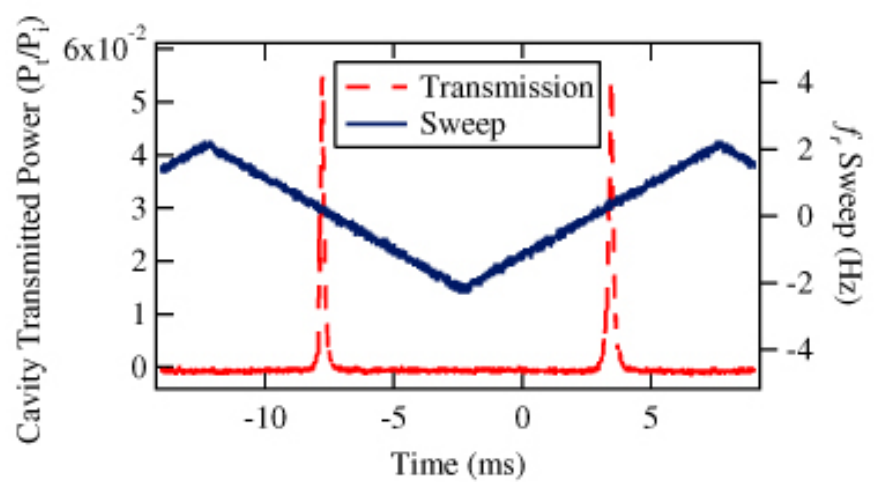

Fig. 7 Cavity transmission for a $100 \mathrm{~nm}$ input spectrum coupled to a $\mathcal{F}=30000$ cavity. Due to low dispersion and optimal cavity/comb matching, the entire $100 \mathrm{~nm}$ incident spectrum becomes resonant with the cavity at nearly the same time during a frequency sweep.

Another advantage of sweeping over the resonance is that it largely avoids intensity noise on the cavity transmitted beam. Under the locked condition, mechanical vibration-induced cavity length fluctuations cause amplitude noise on cavity transmission, which can place a limit on the detection $S / N$ of the transmitted beam. By sweeping at rates larger than the Fourier frequencies of dominant mechanical noise processes, the intensity noise of cavity transmission is drastically reduced.

The final advantage of fast sweeping is the ease of control to maintain the desired coupling between comb and cavity modes. This approach only has to stabilize 
the time at which the comb modes come onto resonance with the cavity modes during the sweep cycle. As a result, a low bandwidth servo acting on the cavity or laser length is sufficient for maintaining a constant sweepbased comb-cavity coupling. An example of this type of servo is given in Fig. 8. The cavity transmission is compared against a preset DC threshold in a comparator, generating a train of pulses that indicate when the comb is resonant with the cavity. These pulses become the 'clock' input for a subsequent D-flip flop. The 'D' input of the flip flop is provided by a TTL synchronization signal for the sawtooth sweep waveform. The output is high when sawtooth is increasing in amplitude and low when it is decreasing. The output of the flip flop can then be integrated to indicate the separation between successive cavity transmission peaks. A DC offset voltage can then be used to lock the separation of successive cavity transmission peaks to any desired value. The value of $f_{o}$ is measured either by the interaction of the comb with the cavity, or by $f-2 f$ interferometry and is then set to a value that optimizes the coupling of the comb to the cavity.

The primary disadvantage of sweep-based coupling is the loss of duty cycle for useful cavity transmission. Making a faster sweep reduces the intensity noise, but the power transmitted through the cavity is also reduced. Therefore it is necessary to choose an optimum sweep rate that maximizes the overall $S / N$ of the cavity transmission by making a compromise between the intensity

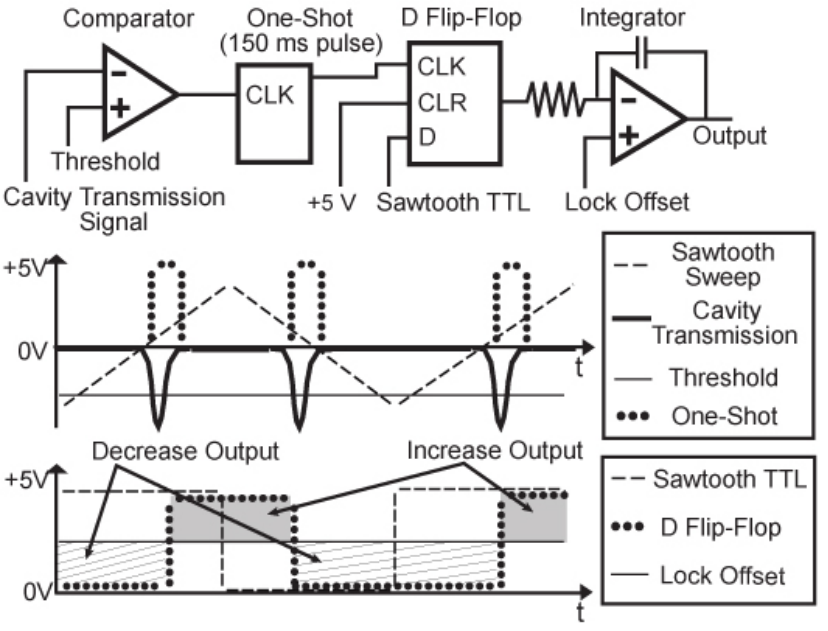

Fig. 8 A schematic of the servo used to maintain the position at which the comb and the cavity come onto resonance during each sweep cycle.

noise and the transmission power. For systems implemented in our lab, a sweep rate of $1-2 \mathrm{kHz}$ is optimal such that the cavity is allowed to build-up intensity for $1 / 10 \tau_{\text {cavity }}$. Of course, the optimal settings depend on the bandwidth of the sweep actuator, and the degree of isolation of the cavity and laser system from mechanical vibrations of their environment. Less mechanical noise allows for a longer intracavity build-up time and higher optical power in the cavity transmitted beam.

\subsection{Precision sweep of laser $f_{r}$}

The final method of coupling the comb to cavity involves purposely mismatching the cavity $F S R$ and the laser $f_{r}$. The comb modes are then swept precisely through successive cavity modes such that the cavity acts as a frequency filter in transmission, allowing one or a handful of comb components to be transmitted at a time. This 
allows serial detection of individual frequency comb components across the comb spectrum [28].

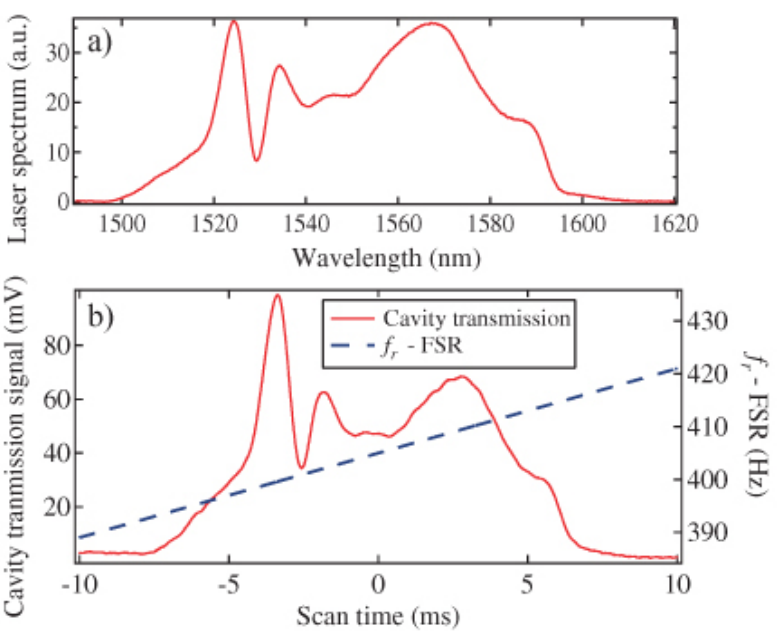

Fig. 9 a) The optical spectrum of a mode-locked $\mathrm{Er}^{+3}$ fiber laser measured with an optical spectrum analyzer. b) The cavity transmission signal as a function of the laser $f_{r}$ detuning form the cavity FSR. The resulting cavity transmission signal recorded over time is not sharply peaked, but rather resembles the original spectrum of the mode-locked laser source.

To illustrate this approach, we demonstrate the measurement of the spectrum of our mode-locked fiber laser using two distinctly different methods (Fig. 9). Fig. 9a displays the spectrum of the mode-locked laser recorded by a traditional optical spectrum analyzer. In comparison, Fig. 9b shows the cavity transmission signal for a detuning between $F S R$ and $f_{r}$ scanned in the range of 390 to $420 \mathrm{~Hz}$. This scan of the laser $f_{r}$ lasted about $20 \mathrm{~ms}$. The resulting cavity transmission signal recorded over this time interval closely resembles the optical spectrum of the mode-locked laser. The optical frequency res- olution and spectral bandwidth provided by the cavity filtering scheme is determined by the laser $f_{r}$, the detuning of the cavity $F S R$ and $f_{r}\left(\delta f=f_{r}-F S R\right)$, and the cavity linewidth $(\Delta \nu)$. The resolution is given by $\Delta \nu$ for $\Delta \nu<\delta f ;$ or $(\Delta \nu / \delta f) f_{r}$ for $\Delta \nu>\delta f$. The first scenario corresponds to the case where only one comb mode is resonant with the cavity at a time while the second case gives the resolution when several adjacent comb modes are simultaneously resonant with the cavity.

The spectral bandwidth that can be achieved in a single scan is determined by $\delta f$ and $f_{r}$. Figure 10 illustrates that if the comb is on resonance with the cavity at a particular frequency, $\delta f$ will initially cause the comb modes to become detuned from the cavity modes in the neighboring spectral region. However, after a frequency interval of $\left(f_{r} / \delta f\right) f_{r}=f_{r}^{2} / \delta f$ the comb will once again become resonant with the cavity modes. This relation can be easily derived by noting that $\Delta N f_{r}=(\Delta N+1) F S R$ $=(\Delta N+1)\left(f_{r}-\delta f\right)$, hence the number of modes contained within the spectral bandwidth is $\Delta N \approx f_{r} / \delta f$.

When more than one frequency is transmitted from the cavity at a time, there is no longer a unique mapping between scan time and the frequency in cavity transmission. To avoid ambiguity in such a case, a dispersive element with a coarse resolution (only need to be better than $\left.f_{r}^{2} / \delta f\right)$ should be placed between the cavity transmission and the detector. To obtain a precise calibration of the cavity transmitted wavelength during a scan of 
$f_{r}$ requires that the frequencies of the cavity modes be known to high precision. For this, the dispersion measurement technique described in Section 3.2 can be used to provide a high precision measurement of the wavelength dependence of the cavity mode frequencies. Figure 10 illustrates the frequency domain picture of cavity filtering of a comb where $f_{r}=100 \mathrm{MHz}, \Delta \nu=3 \mathrm{kHz}$, and $\delta f=1 \mathrm{kHz}$.

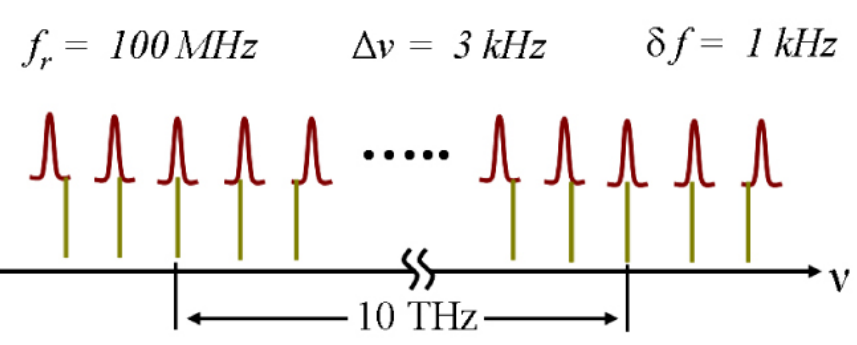

Fig. 10 An example of the resolution (300 MHz) and spectral bandwidth $\left(f_{r}^{2} / \delta f=10 \mathrm{THz}\right)$ provided by cavity filtering for $f_{r}=100 \mathrm{MHz}, \delta f=1 \mathrm{kHz}$, and $\Delta \nu=3 \mathrm{kHz}$.

To achieve the actual cavity-linewidth-limited spectral resolution for $\Delta \nu<\delta f$, the frequency difference between the cavity and comb modes needs to be stabilized to within the cavity linewidth. If the jitter of the cavity or comb modes are larger than the cavity linewidth, then the value of $(\Delta \nu)$ used for determining the resolution should be replaced with the frequency jitter. Since cavities used for sensitive detection can have linewidths in the $\mathrm{kHz}$ range, stabilizing the jitter below this level is straightforward in a research laboratory but could be challenging in field applications.
Compared to the frequency sweep technique for coupling the comb to the cavity, the approach of precision $f_{r}$ scan typically uses slower sweep rates of the comb modes. It is therefore more susceptible to cavity/comb coupling noise arising from mechanical vibrations. Furthermore, this approach takes measurements in a serial manner, prolonging the measurement time. However, this technique does have the benefit of spectrally resolving the cavity transmitted signal without the need for an external spectrometer, a unique feature not shared by the two other coupling techniques.

\section{Measurement schemes}

This Section describes three measurement schemes that can be used to recover intracavity absorption information from the cavity transmitted beams. The two common methods are cavity-enhanced absorption spectroscopy (CEAS) and cavity ring-down spectroscopy (CRDS). An intermediate scheme is transient CEAS.

\subsection{Cavity-enhanced absorption spectroscopy}

CEAS refers to measurements of the intracavity absorption that are made by comparing the power transmitted through the cavity with and without absorption present. These measurements can be performed by either locking the comb to the cavity or by sweeping the comb frequencies over the cavity modes. For the purposes of this section, locked measurements will be referred to as 
steady state CEAS while measurements involving frequency sweep will be referred to as transient CEAS. As will be seen in the following section, the signal to noise ratio achieved in cavity transmission and thus the absorption sensitivity depends slightly on the type of CEAS that is implemented.

For steady state CAES measurments, the comb is locked to the cavity, allowing the intracavity power in each mode to reach steady state after several cavity lifetimes. For a single comb component that is on resonance with a cavity mode, the effect of absorption on the power of the light transmitted through the cavity is given by

$$
\frac{P_{t}(\nu)}{P_{\text {inc }}}=\frac{T_{1} T_{2} e^{-\alpha(\nu) L}}{\left|1-\sqrt{R_{1} R_{2}} e^{-\alpha(\nu) L}\right|^{2}} .
$$

Here $\mathrm{P}_{t}(\nu)$ is the transmitted power, $\mathrm{P}_{i n c}$ is the power incident on the cavity, and $\mathrm{R}_{1,2}$ and $\mathrm{T}_{1,2}$ are the reflectivity and transmission coefficients of the cavity mirrors at frequency $\nu$. $L$ is the cavity length and $\alpha(\nu)$ is the frequency-dependent intracavity absorption 63 64. In the limit that $\alpha(\nu) L \ll 1$ and $R_{1,2} \approx 1$, the change in transmitted power due to absorption $\alpha(\nu)$ can be written as

$$
\frac{\delta P_{t}(\nu)}{P_{\text {inc }}}=\frac{2 F \alpha(\nu) L}{\pi}
$$

Equation 5 illustrates that in the limit of low absorption, steady state CEAS results in an enhancement of the single pass absorption signal by a factor of $2 F / \pi$. Steady state CEAS has the benefit that it is the most sensitive of the intracavity absorption measurement techniques. However, as discussed in the previous section, there are several drawbacks to this technique. The first is the complexity of the comb/cavity servo system since it is necessary to control both the laser $f_{r}$ and $f_{o}$ to high precision. Secondly, intracavity dispersion due to the mirrors and sample gas results in a limited spectral bandwidth over which the comb frequencies and cavity modes are overlapped. Finally, when locking a comb to a high finesse cavity, it is difficult to avoid frequency to amplitude noise conversion, unless a technique similar to NICEOHMS is employed 65 66]. Consequently, the sensitivity benefit of steady state CEAS is often compromised by technical noise.

Transient CEAS, performed by a frequency sweep between the comb and cavity modes, overcomes some of the limitations of steady state CEAS. This scheme is relatively easier to implement due to the relaxed locking requirements and it solves the problem of limited spectral bandwidth encountered when locking the comb to the cavity. The cost of transient CEAS is a reduced sensitivity for absorption measurements.

To perform transient CEAS, the comb modes are made to overlap the cavity modes like they would be for steady state CEAS. However, instead of locking the comb to the cavity, a low modulation amplitude $(<10 \%$ $F S R$ ), fast $(>1 \mathrm{kHz})$ sweep is applied to either the laser $f_{r}, f_{o}$, or the cavity $F S R$. For a single comb component, the effect of intracavity absorption on the transmitted 
power is given by

$$
\frac{P_{t}(\nu)}{P_{\text {inc }}}=T_{1} T_{2}\left|\sum_{n=1}^{\infty} r_{1}^{n-1} r_{2}^{n-1} e^{-(2 n-1) \alpha L / 2} e^{i \phi_{n}(t)}\right|^{2} .
$$

Here $n$ is the number of cavity round trips and $\mathrm{r}_{1,2}$ are the electric field reflection coefficients for the cavity mirrors. The phase term is given by

$$
\phi_{n}(t)=2 \pi(2 n-1)\left(L \frac{\nu(t)}{c}-(n-1) \beta \frac{L^{2}}{c^{2}}\right)
$$

where $\nu(t)$ is the instantaneous frequency of the comb component, and $\beta$ is its frequency sweep rate $67[68$. Besides the lower detection sensitivity, transient CEAS also has the disadvantage that quantitative measurements of the intracavity absorption requires careful calibration. The calibration accuracy is contingent on precise control of the frequency sweep. Maintaining a constant sweep rate is important. A common culprit causing non-constant sweep rates is the hysteresis found in transducers such as piezo electrics. It is therefore necessary to either make measurements only in one sweep direction or determine the actual frequency sweep function and take measurements when the opposing sweep rates are equal.

\subsection{Cavity ring-down spectroscopy}

Another method for making intracavity absorption measurements is cavity ringdown spectroscopy (CRDS) 57 69] 70] 71 72. Light is first injected into the resonant modes of the optical cavity. When the intracavity power in each cavity mode reaches a sufficient level, the light incident on the cavity is switched off. The cavity decay signal is then recorded by a photodiode with a detection bandwidth exceeding that of the cavity decay rate. The power in each cavity mode decays exponentially with a decay constant given by

$$
\tau_{\text {cavity }}=\frac{1}{F S R(\nu) L o s s}=\frac{1}{F S R(\nu)\left(1-R_{1} R_{2} e^{-2 \alpha(\nu) L}\right)} .
$$

Depending on the length and finesse of the optical cavity, this decay time can take on values ranging from tens of nanoseconds to hundreds of microseconds. In the limit that $\alpha(\nu) \ll 1$ and $R_{1,2} \approx 1$, the change in cavity decay time due to absorption $\alpha(\nu)$ can be written as

$$
\frac{\delta \tau_{\text {cavity }}(\nu)}{\tau_{\text {cavity }}}=\frac{F \alpha(\nu) L}{\pi}
$$

For CRDS, the absorption sensitivity in the low absorption limit is exactly half of the steady state CEAS sensitivity. This is due to the fact that for steady state CEAS, the presence of the incident beam suppresses decay of the field from the input mirror. While in CRDS, the field decays through both the input and output mirrors. In the case that the reflectivity of the input and output mirrors are exactly equal, CEAS is twice as sensitive to intracavity absorption as CRDS. In the case of unequal reflectivities, equations 4 and 8 can be used to determine the relative sensitivities.

CRDS has a couple of advantages over the CEAS methods for measuring intracavity absorption. First, since the laser is effectively switched off during the measurement time, cavity ringdown measurements are free of 
laser intensity noise. Also, since the finesse of an optical cavity is typically a very stable quantity that can be measured to high precision, background measurements of the cavity decay rate without any intracavity absorption can be performed relatively infrequently. Hence, more time can be devoted to making absorption measurements rather than calibrating them. The only drawback of cavity ringdown spectroscopy is that it requires fast sampling to record the cavity decay rate. This becomes challenging in broadband applications where hundreds or even thousands of channels must be simultaneously sampled to record an intracavity absorption spectrum.

\subsection{Sensitivity comparison}

To conclude this section we present a comparison of the sensitivity between each of the three measurement schemes. This comparison will be illustrated by the sensitivity of a transient CEAS measurement as a function of the speed at which a comb frequency is swept over the cavity mode. In the limit of an infinitely slow sweep, the sensitivity of transient CEAS approaches that of steady state CEAS. In the limit of a very fast sweep speed, the sensitivity of transient CEAS approaches that of CRDS. Figure 11 shows both the low absorption limit detection sensitivity and the time-integrated fractional transmitted power in transient CEAS as a function of the sweeping speed of the incident laser frequency.

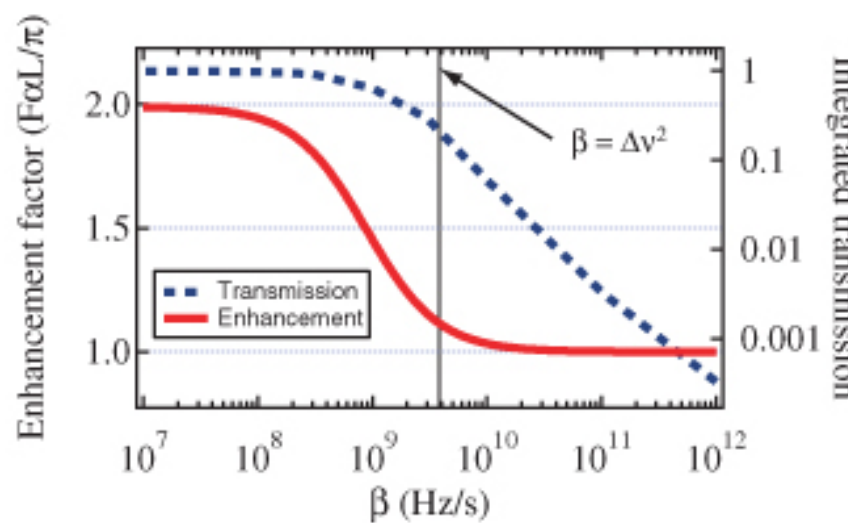

Fig. 11 Absorption sensitivity and integrated power of cavity transmission versus sweep speed $(\beta)$ for transient CEAS. Cavity $F S R=400 \mathrm{MHz}$ and mirror reflectivity $\mathrm{R}=0.9995$ resulting in a cavity linewidth $\Delta \nu=63.7 \mathrm{kHz}$. A time window of $100 \mu$ s was used for integrating the cavity transmitted signal.

For the calculations displayed in Fig. 11, the cavity $F S R=400 \mathrm{MHz}$ and the mirror reflectivity $\mathrm{R}=0.9995$, resulting in a cavity lifetime of $2.5 \mu \mathrm{s}$. A time window of $100 \mu$ s was chosen for integrating the cavity transmitted signal. The center wavelength was $1.525 \mu \mathrm{m}$ and the intracavity absorption was $10^{-8} \mathrm{~cm}^{-1}$. At slow sweep speeds, it takes a comb frequency many cavity lifetimes to scan across the cavity linewidth. Under these conditions, the intracavity power nearly reaches the steady state value and cavity transmitted beam power is $P_{\alpha}=$ $P_{o}(1-2 F \alpha L / \pi)$. Here $P_{o}$ is the cavity transmitted power with no intracavity absorption. For a fast sweep speed, the comb frequency scans across the cavity resonance in less than a cavity lifetime. In this case the peak intracavity power is much smaller than the steady state 
value, and the majority of the cavity transmitted signal takes the form of an exponential decay. Since the time integral of an exponentially decaying signal is proportional to its decay time, the change in the integrated cavity transmission due to absorption is just proportional to the change in the cavity ringdown time, thus linking transient CEAS to CRDS. When the frequency sweeping speed $(\beta)$ is such that the comb frequency traverses the cavity linewidth in one or a few cavity lifetimes $\left(\beta \approx \Delta \nu^{2}\right)$, changes in the cavity transmission due to intracavity absorption arise from a combination of steady state CEAS and CRDS effects.

In practice, it is usually advantageous to sacrifice the factor of 2 enhancement associated with steady state CEAS and work in the regime of fast sweeping. Doing so allows us to avoid relatively low frequency mechanical noise present in both the laser and the passive optical cavity. The gain in $S / N$ by making detection at Fourier frequencies where mechanical noise is low is typically much greater than the factor of 2 gained via steady state detection.

\section{Detection schemes}

In the previous section we have outlined a few strategies for making intracavity absorption measurements. In this section we focus on broad-bandwidth detection schemes that can be implemented to provide frequency-resolved and massively parallel detection channels for the intracavity absorption. Depending on particular applications, different detection schemes can provide uniquely optimized performances.

\subsection{Photodiode array or CCD detection}

The simplest way to perform parallel detection of a broadband cavity transmission signal is to use a dispersive element such as a diffraction grating followed by imaging optics and a multi-element photodetector. The detector can take the form of a photodiode array or a CCD camera. In this configuration, the final detection resolution is determined by the spectral resolving power of the grating and the imaging resolution on the detector array. Grating spectrometers typically provide resolutions on the order of a few to many tens of gigahertz. The overall spectral bandwidth that can be recorded by the detector in a single shot is limited by the size of the detector array and the area over which a flat field of imaging can be maintained. Single shot spectral bandwidths range from hundreds of gigahertz to several terahertz. For a fixed detector size with $n$ resolvable detection channels and a resolution of $\delta \nu$, the spectral bandwidth that can be recorded in a single shot is $n \delta \nu$. This relationship illustrates the compromise that must be made between single shot bandwidth and resolution that is encountered when designing a broadband detection system.

Another issue that arises from multichannel detectors is the rate at which the array can be read out. Depending on the detector size and the type of a multiplexer used for sampling, read-out rates in the tens of $\mathrm{Hz}$ to tens of 
$\mathrm{kHz}$ range can be achieved. While the later provides fast frame acquisitions for signal averaging, it is still too slow to directly observe cavity ringdown decays. Most cavities require a sampling rate of around $1 \mathrm{MHz}$ to perform accurate ringdown measurements. To simultaneously sample ringdown signals from a large-numbered photodiode array, we have developed an electronics architecture that is capable of sampling and recording ringdown events from an arbitrarily large photodiode array at rates that can easily exceed $1 \mathrm{MHz}$.

A block diagram of the electronics architecture is shown in Fig. 12. A large photodiode array, consisting of hundreds to thousands of detector elements, is first divided into sub-arrays of 10 to 50 elements that detect adjacent portions of the optical spectrum. The photodiode array in Fig. 12 represents one of these sub-arrays. The

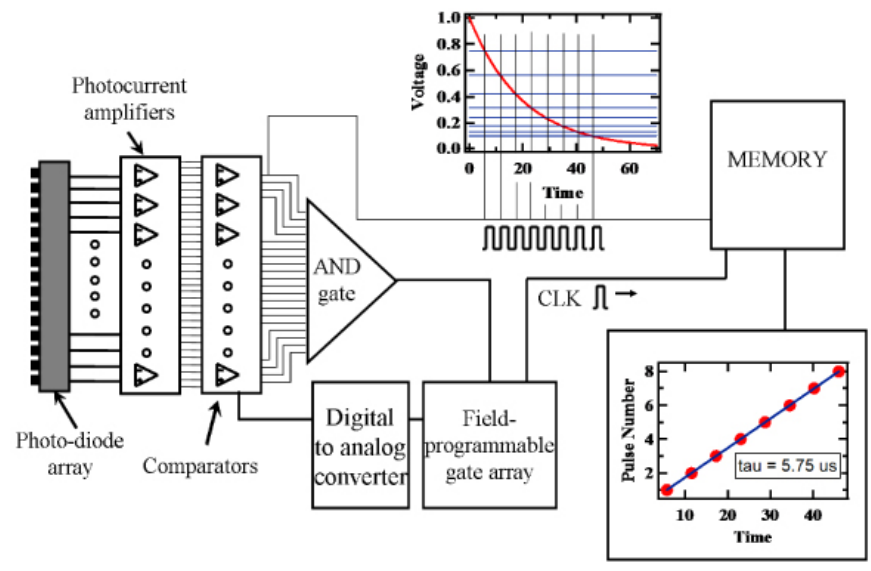

Fig. 12 A circuit for parallel readout of cavity ringdown signals from a photodiode array.

current from each element in the sub array is converted to a voltage by a bank of transimpedance amplifiers.
Next, a bank of comparators is used to compare these photo-voltages against a known reference voltage that is set by a digital-to-analog converter (DAC). The reference voltage is chosen to be a fraction of the peak voltage recorded by one of the detector elements in the subarray. As the intracavity field decays, each photo-voltage drops below the reference voltage and the corresponding comparator emits a pulse that is recorded by a clocked memory chip. The comparator pulse is also sent to the input of an AND gate. When each signal drops below the DAC voltage, the output of the AND gate switches, instructing the DAC to set a new reference voltage. The subsequent reference voltages are chosen to be a power law series such that an exponentially decaying ringdown signal will produce a series of comparator pulses that are equally spaced in time. This sequence continues until all signals drop below the lowest reference voltage. During this process, a field programmable gate array (FPGA) performs a number of operations to ensure that switching of reference voltages occurs at the appropriate times and that the ringdown signal for each channel is recorded correctly. When the comparator pulse arrival data is retrieved from the memory chip, a simple linear fit of the arrival time of the comparator pulses versus pulse number reveals the ringdown time of that channel. So far, only a small prototype of this circuit consisting of four channels has been built and tested, but the scalability of this architecture will allow for hundreds to thousands of 
channels to be simultaneously sampled with small additional effort.

\subsection{VIPA spectrometer}

One way to overcome the compromise between spectral bandwidth and resolution is to use a virtually imagedphased array (VIPA) spectrometer (Fig. 13) [73] 25] 29]. This spectrometer uses a tilted solid thin Farby-Perot etalon, oriented orthogonally to a diffraction grating that is placed after the etalon, to disperse the cavity transmitted beam into a two-dimensional pattern, with each spatial element corresponding to a unique comb frequency. The 2D spatial pattern of the comb is then imaged onto

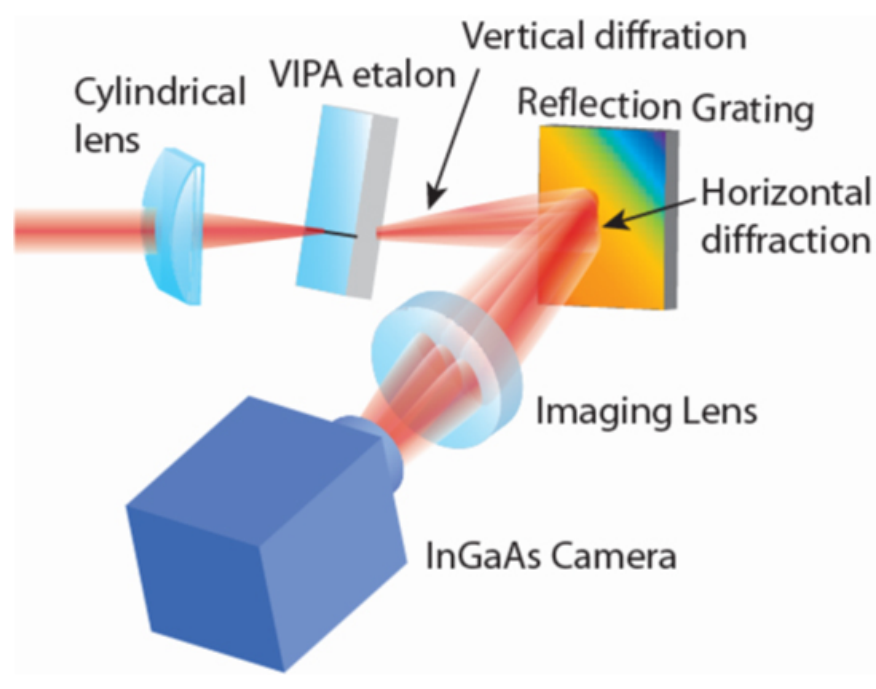

Fig. 13 A schematic of the VIPA spectrometer.

a CCD camera to record the frequency-resolved power of the cavity transmission. The angular dispersion of the VIPA etalon can be 30 to 40 times greater than the dispersion of an optical grating. As a result, light dispersed along the tilt of etalon provides a high spectral resolu- tion, while light dispersed along the orthogonal direction by the grating acts to resolve the different mode orders transmitted through the VIPA etalon.

Figure 14 shows a typical VIPA absorption image of the rovibrational spectrum of $\mathrm{CO}_{2}$ centered at $1.609 \mu \mathrm{m}$. Each vertical fringe contains nearly two FSR mode orders of the etalon transmission, which has been dispersed and resolved in the horizontal direction by the grating. To resolve the vertical fringes in a VIPA spectrometer, the grating must have a resolution that is higher than the $F S R$ of the VIPA etalon.

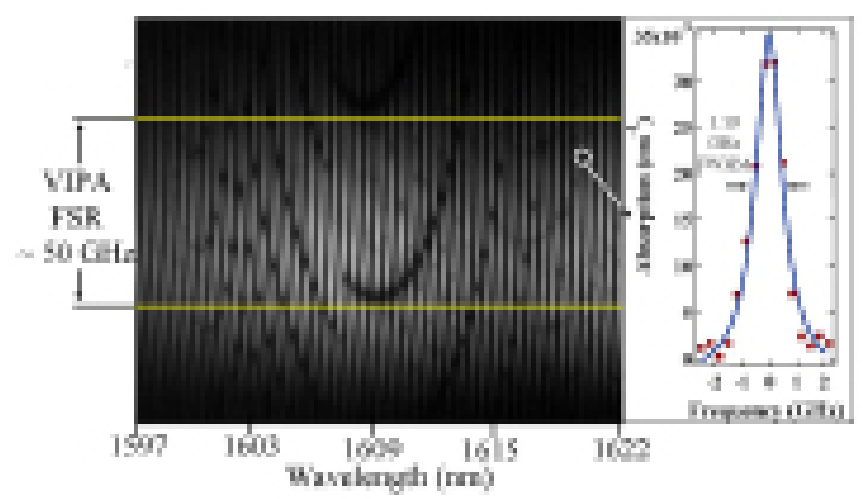

Fig. 14 An image of a rotational spectrum of a $\mathrm{CO}_{2}$ vibration overtone centered at $1.609 \mu \mathrm{m}$ recorded with the VIPA spectrometer. The image contains $25 \mathrm{~nm}$ of spectrum with $833 \mathrm{MHz}$ resolution. The panel to the right shows the P13 line of the (011) to (311) vibration overtone band, illustrating the resolution.

In Fig. 14, the VIPA etaon has a FSR of $50 \mathrm{GHz}$ while the 1100 lines $/ \mathrm{mm}$ grating imaged by a $f=20 \mathrm{~cm}$ lens is capable of resolving $25 \mathrm{GHz}$. The camera recording the $2 \mathrm{D}$ pattern has dimensions $256 \times 320$ pixels with a pixel pitch of $25 \mu \mathrm{m}$. The diffraction limited spot size 
of the beam imaged on the camera is $50 \mu \mathrm{m}$ such that each resolvable frequency component in the spectrometer focal plane is sampled by 4 pixels of the camera. The size of the camera, the grating dispersion and the imaging lens focal length lead to a $25 \mathrm{~nm}$ spectral bandwidth captured in a single image. Since there are 120 vertical pixels contained in one $F S R$ of the recorded image, the resulting spectral resolution is roughly equal to (50 $\mathrm{GHz}) /[(120$ pixels $) /(2$ pixel resolution $)]=833 \mathrm{MHz}$. To accurately calculate the resolution and wavelength as a function of the position on the camera requires the generalized grating equation for the VIPA [74]. In the example presented here, more than 3500 spectral channels are acquired simultaneously, far more than that could be achieved in one dimension and with significantly higher resolution.

To extract a traditional absorption spectrum from the recorded VIPA images requires a modest amount of image processing. A flow diagram of the spectral recovery process is presented in Fig. 15, where a pair of $256 \mathrm{x}$ 320 pixel images are converted into a $25 \mathrm{~nm}$ absorption spectrum. The first step is to locate the nearly vertical fringes produced within the picture. This is done using the reference image, which is collected without intracavity absorption. Fringes are collected by an algorithm that begins at the peak of each fringe at the middle of the picture (i.e. row 128) and 'walks' along each fringe up and down to find the peak of each fringe within the entire picture frame. The intensity of each fringe is converted into a column vector forming an array that contains frequency resolved cavity transmitted power information.
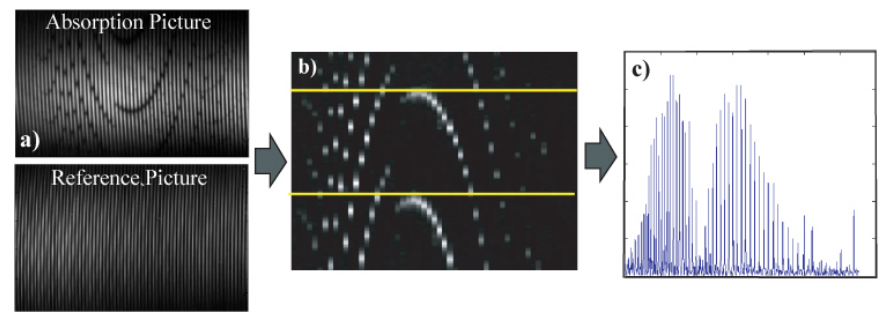

Fig. 15 Generation of an absorption spectrum from VIPA images. a) absorption and reference images are recorded. b) The nearly vertical fringes in both the reference and absorption images are collected into column vectors and processed according to $\left(P_{r}-P_{a}\right) / P_{r}$ to form a relative power array. The relative power array is used to find the VIPA $F S R$, indicated by the area between the solid lines. c) The fringe intervals between the solid lines are then collected and arranged endwise to form a traditional absorption spectrum.

To correct for possible drifts in the cavity transmitted power between the acquisition of the absorption and reference pictures, an area of low absorption in the absorption fringe array is equalized in power to the reference fringe array. The two fringe arrays are subtracted and then divided by the reference fringe array, resulting in a power-normalized fringe array shown in Fig. 15b.

The FSR interval of the VIPA, shown as the interval between the two solid horizontal lines in Fig. 15b, is determined from the recognition of the repetitive patterns of absorption peaks in the power-normalized fringe array. All unique information about the molecular absorption is 
contained within this interval. Once this interval is determined, the area of the normalized fringe array between the horizontal lines is unwrapped into a one dimensional spectrum of relative power versus wavelength. Here the generalized grating equation for the VIPA can be used to obtain a high precision mapping of the position of each wavelength on the normalized fringe array. Finally, the cavity enhancement equations from Section 5 are used to convert changes in cavity transmitted power to their corresponding values of intracavity absorption.

\subsection{Precision scan of $f_{r}$ and cavity filtration}

Another approach for achieving high spectral resolution over a large spectral window is cavity-filtered comb detection. Unlike previously mentioned approaches that use optical dispersers to analyze the cavity transmission, the cavity-filtered detection uses the frequency response of the cavity itself to allow only one spectral component of the frequency comb to be transmitted through the cavity at a time. In this Section we present an example of cavity-filtered detection. The cavity frequency filtration is discussed in Section 4.3. The results presented here are based on a $f_{r}=99 \mathrm{MHz}$ erbium fiber modelocked laser comb, a detection cavity with a FSR of 396 $\mathrm{MHz}\left(F S R=4 \times f_{r}\right)$ and a finesse of 6300 at the detection wavelength $1.525 \mu \mathrm{m}$. The intrinsic cavity linewidth is $63.5 \mathrm{kHz}$.

To use the cavity-filtration approach for frequencyresolved detection of cavity transmission, it is necessary to precisely control the frequencies of both the cavity and comb modes. For the measurements presented here, the frequency stabilization scheme is shown in Fig. 16. The cavity is stabilized by locking a particular cavity resonance (and thus the cavity length) to a frequencystabilized Nd:YAG laser at $1064 \mathrm{~nm}$. The cavity length is

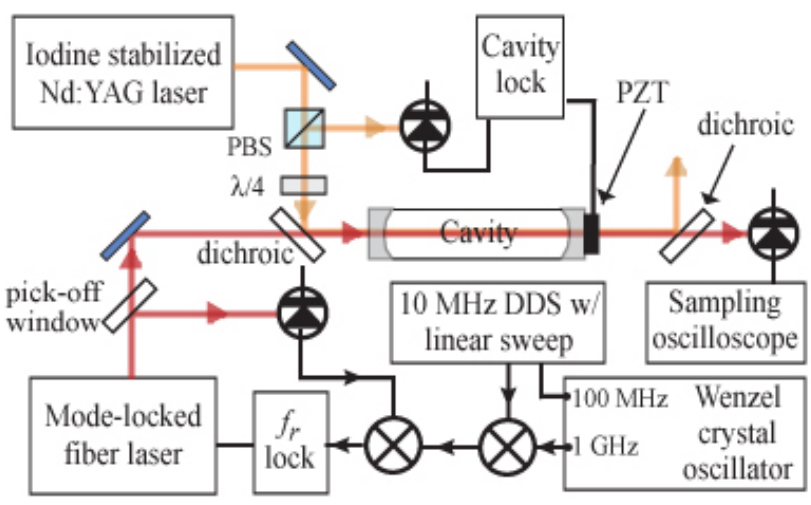

Fig. 16 Schematic for the cavity filtration measurement setup. An iodine stabilized Nd:YAG laser $(1064 \mathrm{~nm})$ is used to stabilize the cavity frequencies via a piezo electric actuator that controls the cavity length. The $10^{\text {th }}$ harmonic of the laser $f_{r}$ is locked to a microwave signal that is the mixing product of a $1 \mathrm{GHz}$ Wenzel oscillator and a $10 \mathrm{MHz}$ DDS. To scan a spectrum, the DDS frequency is set such that the laser $f_{r}$ is detuned from the cavity FSR. The DDS frequency is then linearly scanned, resulting in a time-dependent output frequency for the cavity transmission.

stabilized to $\lambda / 3200 \mathrm{RMS}$. For $F S R \sim 400 \mathrm{MHz}$, this corresponds to stabilization of a cavity resonance frequency to within $250 \mathrm{kHz}$. To stabilize the relative frequency between the cavity and the comb, the tenth harmonic of the laser $f_{r}$ is locked to the frequency difference of a $1 \mathrm{GHz}$ low-phase-noise crystal oscillator and a $10 \mathrm{MHz}$ auxil- 
iary RF signal from a direct digital synthesizer (DDS).

The time base of the DDS is slaved to the same lowphase-noise crystal oscillator. The DDS is used to generate a high precision linear frequency ramp that sweeps the comb frequencies over the cavity modes. The DDS makes a $500 \mathrm{~Hz}$ ramp in steps of $23 \mathrm{mHz}$, each lasting $10.2 \mu \mathrm{s}$ for a total sweeping time of $0.22 \mathrm{~s}$.

To illustrate the utility of this scheme for frequencyresolved detection of cavity transmission, the cavity was backfilled with 100 mTorr of $\mathrm{C}_{2} \mathrm{H}_{2}$ and $500 \mathrm{~Hz}$ scans were taken at detunings of $\delta f=f_{r}-F S R=2.5 \mathrm{kHz}$ and $5 \mathrm{kHz}$. Figure 17 shows the recovered spectral features as a result of these scans. Since the cavity modes were stabilized to within $250 \mathrm{kHz}$ (effective $\Delta \nu$ ), the $2.5 \mathrm{kHz}$ detuned scan resulted in a resolution of $(\Delta \nu / \delta f) \times f_{r}=$ $10 \mathrm{GHz}$. The available spectral bandwidth for this scan is $f_{r}^{2} / \delta f=3.92 \mathrm{THz}$, or $30 \mathrm{~nm}$ at $1.525 \mu \mathrm{m}$. Figure 17a shows $25 \mathrm{~nm}$, or $83 \%$ of the available scan width, with the $\mathrm{C}_{2} \mathrm{H}_{2}$ absorption spectrum clearly extracted in Fig. 17b. For the $5 \mathrm{kHz}$ scan, $\delta f$ was increased by a factor of 2 and consequently the resolution increased and the bandwidth decreased by the same factor of 2 . This leads to a 5 GHz-resolution spectrum shown in Fig. 17c.

The variations in the baselines of these two scans arise from a combination of cavity/comb coupling noise and absorptions due to unresolved hot bands of $\mathrm{C}_{2} \mathrm{H}_{2}$ that occupy the same spectral region as the first overtone of the asymmetric C-H stretch.
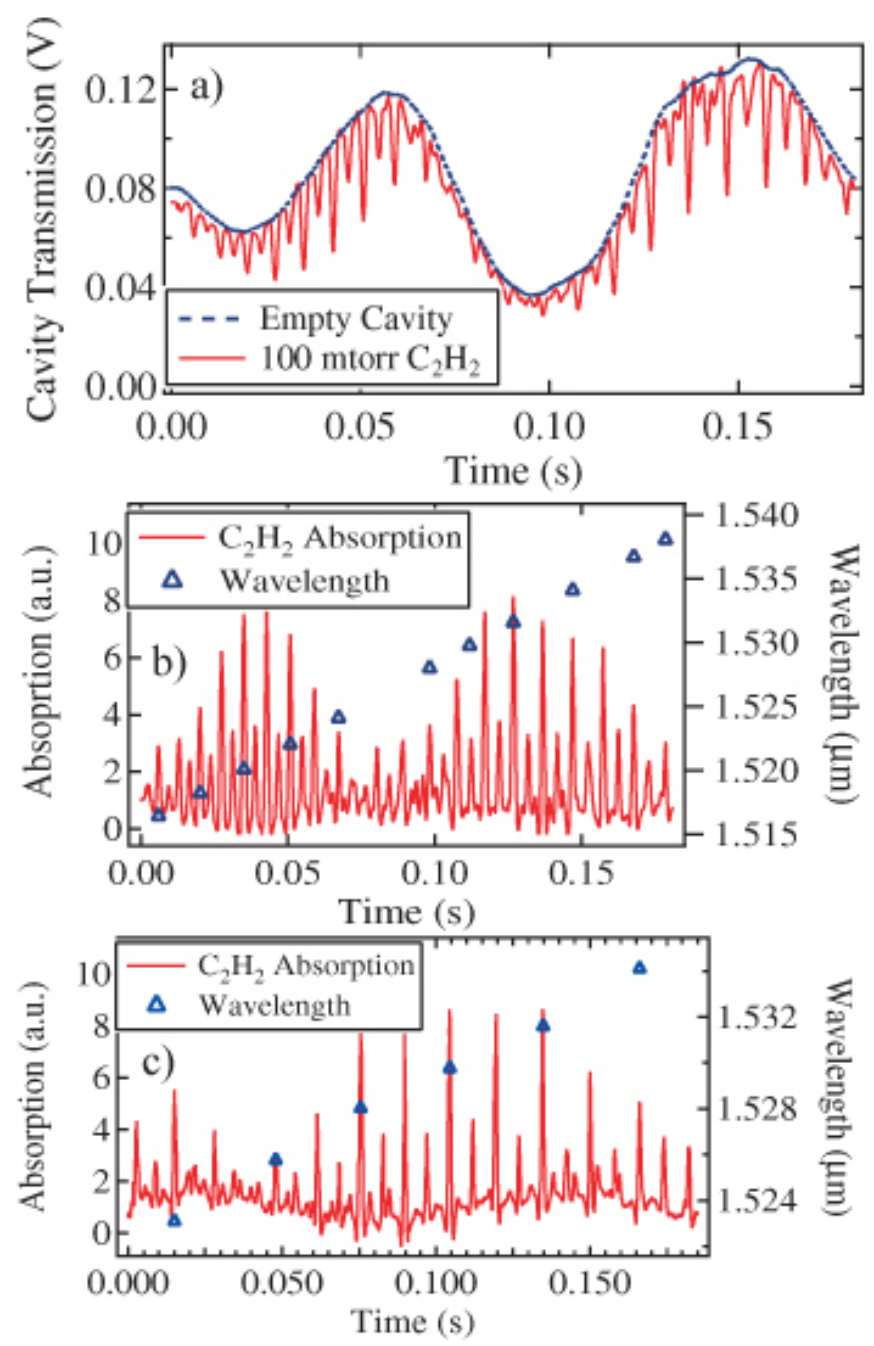

Fig. 17 a) The cavity transmission signal with and without 100 mTorr of $\mathrm{C}_{2} \mathrm{H}_{2}$ inside the cavity for a $2.5 \mathrm{kHz}$ detuning of the laser $f_{r}$ from the cavity $F S R$. b) The $\mathrm{C}_{2} \mathrm{H}_{2}$ absorption spectrum extracted from a) showing $25 \mathrm{~nm}$ of spectrum with $10 \mathrm{GHz}$ resolution. c) A second sweep where $f_{r}$ is detuned 5 $\mathrm{kHz}$ from the cavity $F S R$ resulting in a $12.5 \mathrm{~nm}$ spectrum with $5 \mathrm{GHz}$ resolution.

A couple of options exist to achieve higher resolution capable of resolving these lines. The first and the most intuitive approach is to increase the detuning $\delta f$ and perform scans of higher resolution with narrower spectral bandwidth. The second and more elegant method was 
recently demonstrated by Gohle et al. [28. This method

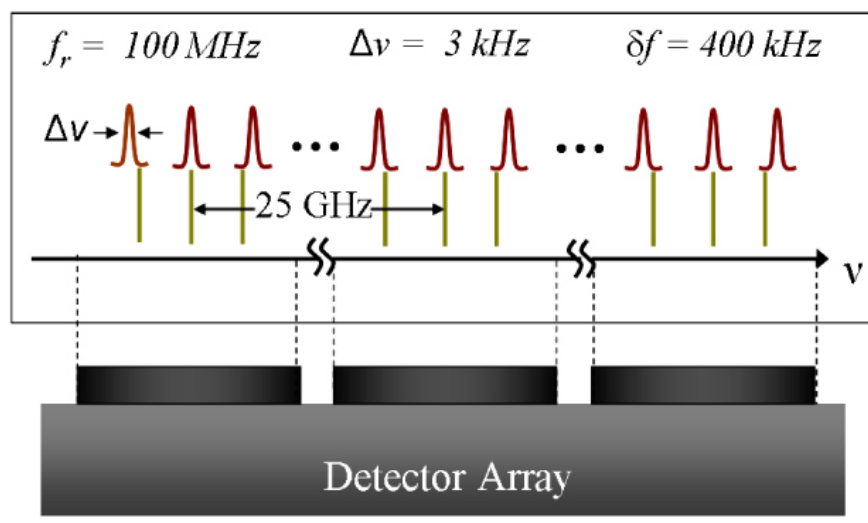

Fig. 18 An example of how the comb/cavity detuning $(\delta f)$ can be selected to match the cavity-filtered spectral bandwidth to the corresponding spatial period of a multichannel spectrometer in cavity transmission.

combines cavity filtering with a grating spectrometer in cavity transmission to achieve high resolution and large spectral bandwidth in a single system. In a sense, this scheme shares the same spirit as the VIPA spectrometer, where a high resolution element (etalon) is combined with a low resolution grating. Whereas the VIPA scheme uses a second spatial dimension to provide high resolution, cavity filtration uses temporal scan of the comb freuqnecies. Fig. 18 shows a schematic of how the cavity filtration and spatial dispersion can work together to provide high resolution and large spectral bandwidth. The detuning $\delta f$ is chosen to match the resolution of the grating spectrometer to the spectral bandwidth of cavity filtration. In doing so, each resolvable unit of the spectrometer contains a single comb component at each step during the frequency scan. For the example shown in Fig. 18, each scan covers 250 cavity modes requiring a scan time of at least 250 cavity lifetimes for single comb mode resolution. For typical cavities, 250 cavity lifetimes corresponds to a scan time of one to tens of milliseconds.

\section{Applications}

The final Section presents a series of CE-DFCS measurements, beginning with initial demonstrations of these techniques followed by more advanced and recent measurements. This overview is meant to highlight the general capabilities of these systems as well as the recent technological advances that have allowed significant simplifications of experimental setups. The measurements presented here include trace detection of room temperature gas samples, breath analysis, and detection and characterization of cold molecules in supersonic jets.

\subsection{Initial demonstrations}

Gherman and Romanini performed broadband CEAS using a Ti:sapphire mode-locked laser to probe the high overtone spectrum of $\mathrm{C}_{2} \mathrm{H}_{2}$ at $860 \mathrm{~nm}$ [26. This system performed transient CEAS measurements using a cavity with a finesse of $\mathrm{F}=420$ and achieved an absorption sensitivity of $2 \times 10^{-7} \mathrm{~cm}^{-1} \mathrm{~Hz}^{-1 / 2}$. A high resolution grating spectrometer and a CCD camera were implemented in cavity transmission to provide $14 \mathrm{~nm}$ of single shot bandwidth with a resolution of $6 \mathrm{GHz}$. 
Thorpe et al. demonstrated frequency-comb-based CRDS distance between the mirror and CCD. Finally, the deusing a Ti:Sapphire laser-based frequency comb at 800 nm to probe the high overtone spectrum of $\mathrm{C}_{2} \mathrm{H}_{2}, \mathrm{H}_{2} \mathrm{O}$, $\mathrm{NH}_{3}$, and a week forbidden electronic transition in $\mathrm{O}_{2}$ [27. An example of these measurements is provided in Fig. 19. This system used a $\mathcal{F}=3300$ cavity and cavity ringdown detection to achieve an integrated absorption sensitivity of $10^{-8} \mathrm{~Hz}^{-1 / 2}\left(2.5 \times 10^{-10} \mathrm{~cm}^{-1} \mathrm{~Hz}^{-1 / 2}\right)$. In

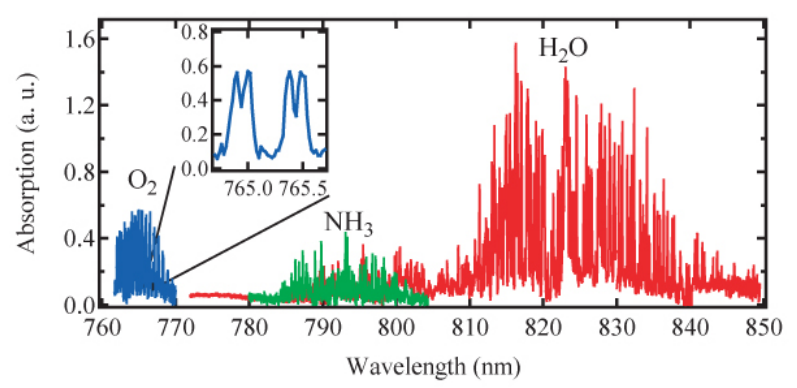

Fig. 19 The spectra of $\mathrm{H}_{2} \mathrm{O}, \mathrm{NH}_{3}$, and $\mathrm{O}_{2}$ from $760 \mathrm{~nm}$ to $850 \mathrm{~nm}$

cavity transmission a grating spectrometer and CCD were implemented to record $20 \mathrm{~nm}$ of single shot spectra with $25 \mathrm{GHz}$ resolution. To achieve adequate time resolution of the ringdown signals, a rotating mirror was installed in the grating spectrometer such that time decay of the cavity transmitted power was streaked across the vertical columns of the CCD 75]. Using this technique the cavity decay signal recorded by the CCD exhibits decaying power as a function of vertical position on the detector. The decay versus position signal is converted to decay versus time using the mirror rotation rate and the cay signal is fit to extract the cavity ringdown time. Each column of the CCD simultaneously records a decay signal at a different wavelength, resulting in a single-shot, broad bandwidth ringdown spectrum.

While these initial systems provide good sensitivities for the study of high overtone spectra, the weak transition strengths of high overtones make them not very desirable for applications such as trace detection. Also, the resolution of the initial systems was too low to resolve the Doppler linewidths of room temperature gases and therefore limited the sensitivity of molecular concentration measurements. In response to these limitations, recent work of Thorpe et al. at $1.5 \mu \mathrm{m}$ with $\mathrm{Er}^{+} 3$ fiber laser-based frequency combs aided by high resolution spectrometers in cavity transmission has drastically increased the detection sensitivity and spectral resolution available with CE-DFCS systems [29].

\subsection{Trace detection at $1.5 \mu \mathrm{m}$}

The trace gas measurements presented here were made with an $\mathrm{Er}^{+3}$ fiber comb with $f_{r}=99 \mathrm{MHz}$. The laser provided a $100 \mathrm{~nm}$ spectrum from 1.5 to $1.6 \mu \mathrm{m}$ which could be shifted to 1.6 - $1.7 \mu \mathrm{m}$ using a Raman shifting amplifier [76]. The comb was coupled to a FSR $=99 \mathrm{MHz}$ and $\mathcal{F}=30000$ cavity via dithering and transient CEAS measurements were performed. A VIPA spectrometer was used in cavity transmission to achieve a 25-nm single shot bandwidth and $800 \mathrm{MHz}$ resolution. 
For these measurements, there are several effects to consider in order to optimize the spectrometer performance. The equation that relates the absorption coefficient measured by the spectrometer to the gas concentration is $\alpha(\nu)=n S_{i} g(\nu)$. Here, $\alpha$ is the absorption per unit length $\left(\mathrm{cm}^{-1}\right), n$ is the gas concentration $\left(\mathrm{cm}^{-3}\right)$, $S_{i}$ is the line intensity $(\mathrm{cm})$, and $g(\nu)$ is the lineshape (cm). The values for $S_{i}$ can be measured, found, or inferred from a variety of references 77 [78. However, the lineshape depends on the environmental conditions of the measurement. In particular, choosing the appropriate intra-cavity gas pressure is important for maximizing the molecular absorption while not diminishing spectral resolution via collision broadening.

The gases that were measured and analyzed in our $1.5 \mu \mathrm{m}$ CE-DFCS system include: $\mathrm{CO}, \mathrm{CO}_{2}, \mathrm{C}_{2} \mathrm{H}_{2}, \mathrm{C}_{2} \mathrm{H}_{4}$, $\mathrm{CH}_{4}$, and $\mathrm{NH}_{3}$. Parts of the recorded spectra for each of these gases are shown in Fig. 20 and Fig. 21. Since these trace gases are measured in a host gas of primarily $\mathrm{N}_{2}$, the air broadening coefficient adequately describes the observed collision broadening. For measurements presented in this Section, an intracavity gas pressure of 250 Torr was chosen such that the average linewidth for a gas under measurement was $1.8 \mathrm{GHz}$, resolvable by the $800 \mathrm{MHz}$ resolution of our system.

For the molecules listed above, the spectral window between $1.5 \mu \mathrm{m}-1.7 \mu \mathrm{m}$ contains more than 10,000 individual lines for measurements at $296 \mathrm{~K}$. To handle this large amount of information, we created a database

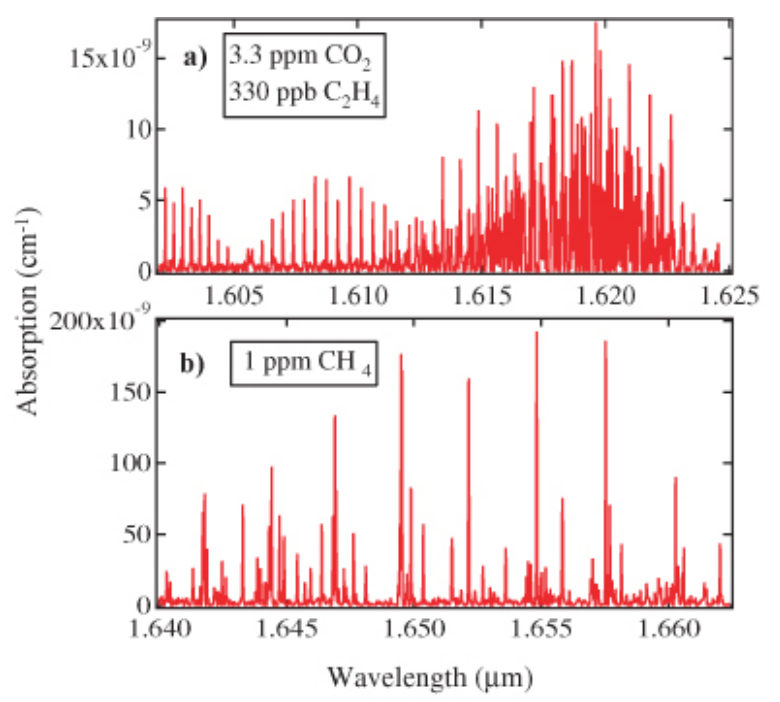

Fig. 20 The rovibrational overtone spectra of a) 330 parts per billion (ppb) $\mathrm{C}_{2} \mathrm{H}_{4}$ and $3.3 \mathrm{ppm} \mathrm{CO}_{2}$ and b) 1 ppm $\mathrm{CH}_{4}$.

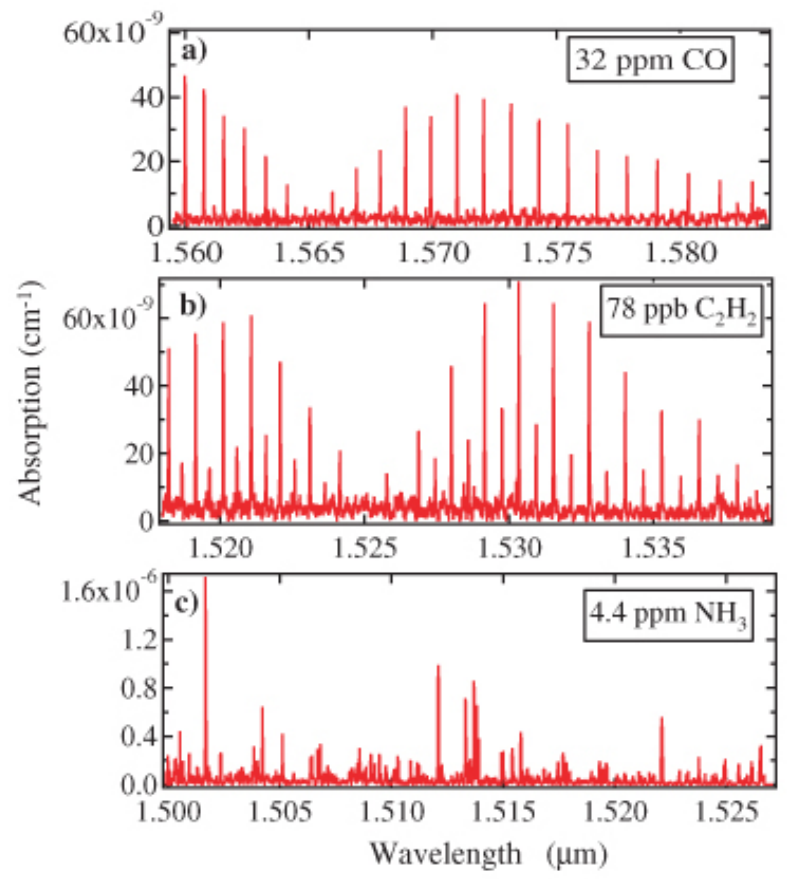

Fig. 21 The rovibrational overtone spectra of a) $32 \mathrm{ppm}$ $\mathrm{CO}$, b) $78 \mathrm{ppb} \mathrm{C}_{2} \mathrm{H}_{2}$, and c) $32 \mathrm{ppm} \mathrm{CO}$, and c) $4.4 \mathrm{ppm}$ $\mathrm{NH}_{3}$.

containing the center frequency, line intensity, and the air-broadening coefficient of more than $60 \%$ of the existing lines. This database was compiled from a variety of sources $77,78,6$. Using this database, gas con- 
Table 1 Spectral location and minimum detectable concentration (MDC) of measured molecules using the $1.5 \mu \mathrm{m} \mathrm{CE}$ DFCS.

\begin{tabular}{lll} 
Molecule & Wavelength $(\mu \mathrm{m})$ & MDC \\
\hline $\mathrm{CO}$ & $1.56-1.62$ & $900 \mathrm{ppb}$ \\
$\mathrm{CO}_{2}$ & $1.56-1.62$ & $770 \mathrm{ppb}$ \\
$\mathrm{CH}_{4}$ & $1.63-1.69$ & $10 \mathrm{ppb}$ \\
$\mathrm{C}_{2} \mathrm{H}_{2}$ & $1.51-1.54$ & $3 \mathrm{ppb}$ \\
$\mathrm{C}_{2} \mathrm{H}_{4}$ & $1.61-1.65$ & $33 \mathrm{ppb}$ \\
$\mathrm{NH}_{3}$ & $1.50-1.55$ & $15 \mathrm{ppb}$ \\
\hline
\end{tabular}

centrations were determined for lines of interest from a recorded spectrum by performing a modified Voigt fit that also included the resolution of the VIPA spectrometer. By finding the value of the instrument resolution that provided the best fit to the experimentally recorded lineshapes, an instrument resolution of $800 \mathrm{MHz}$ was experimentally verified.

Calibration of the spectrometer was performed using three gas mixtures containing a total of four target gases consisting of 10 parts per million (ppm) $\mathrm{CO}_{2}, 1$ ppm $\mathrm{CH}_{4}, 1$ ppm $\mathrm{C}_{2} \mathrm{H}_{4}$, and 1 ppm $\mathrm{NH}_{3}$, all mixed with balanced $\mathrm{N}_{2}$. The accuracy of trace gas concentrations was specified to within $1 \%$ of the total concentration by a commercial supplier of standard gas. Since the spectral lines from these four molecules cover the entire spectral region from $1.5 \mu \mathrm{m}$ to $1.7 \mu \mathrm{m}$, we were able to verify the calibration of the absorption loss provided by the cavity transmission equation (eq. 6) for the entire spectral region. Finally, we made mixtures of $\mathrm{CO}$ and $\mathrm{C}_{2} \mathrm{H}_{2}$ by mixing pure samples of these gases with $\mathrm{N}_{2}$ to determine the minimum detectable concentrations of these gases. These results, along with their detection wavelengths, are listed in Table 1.

\subsection{Isotope ratio measurements}

A powerful demonstration of the sensitivity, dynamic range, and resolution of our spectrometer can be made via isotope measurements. During the course of measuring the spectra of $\mathrm{CO}, \mathrm{CO}_{2}, \mathrm{C}_{2} \mathrm{H}_{2}, \mathrm{H}_{2} \mathrm{O}$, and $\mathrm{CH}_{4}$, we recorded spectral regions that contain multiple isotopes of all of these molecules. Figure 22 shows the spectra of $\mathrm{CO}$ and $\mathrm{C}_{2} \mathrm{H}_{2}$ containing multiple isotopes. For both
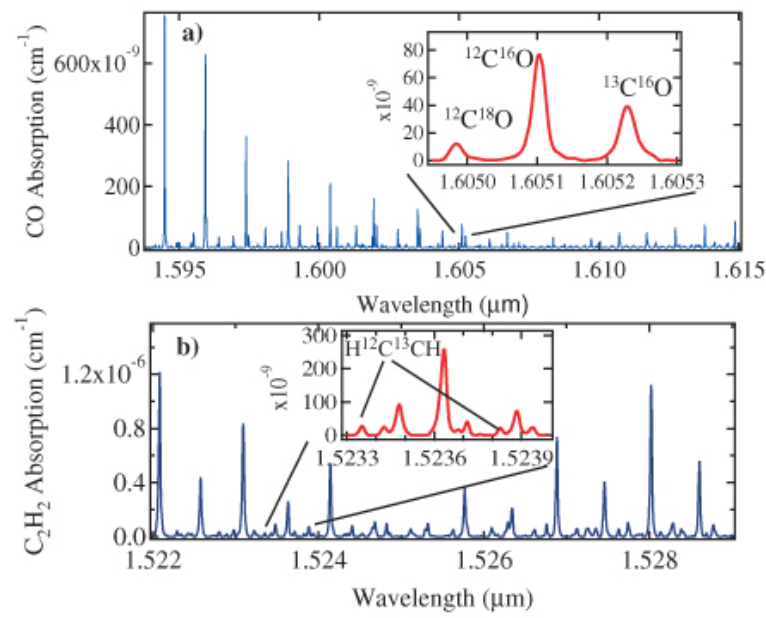

Fig. 22 a) The isotope spectrum of CO near $1.605 \mu \mathrm{m}$ at a $\mathrm{CO}$ concentration of 4.5 parts per thousand. b) The isotope spectrum of $\mathrm{C}_{2} \mathrm{H}_{2}$ near $1.524 \mu \mathrm{m}$ at a $\mathrm{C}_{2} \mathrm{H}_{2}$ concentration of $2.2 \mathrm{ppm}$. The unlabeled lines in the $\mathrm{C}_{2} \mathrm{H}_{2}$ spectrum are ${ }^{12} \mathrm{C}_{2} \mathrm{H}_{2}$ while the labeled lines are $\mathrm{H}^{12} \mathrm{C}^{13} \mathrm{CH}$.

$\mathrm{CO}$ and $\mathrm{C}_{2} \mathrm{H}_{2}$, the relative abundances of the isotopes 
measured by our system matched those found in the HI-

TRAN database to within $0.4 \%$. We also estimated the minimum concentration of $\mathrm{CO}$ and $\mathrm{C}_{2} \mathrm{H}_{2}$ that would allow detection of the second most abundant isotope. These concentrations were 200 ppm for CO and 200 ppb for $\mathrm{C}_{2} \mathrm{H}_{2}$.

The ability to accurately measure molecular isotope ratios has found important applications in many fields of science. For instance, doctors use stable isotope ratios of ${ }^{13} \mathrm{CO}_{2}$ on the breath of patients to detect $\mathrm{He}$ liobacter Pylori for diagnosing ulcers [79]. Geologists use stable isotopes of $\mathrm{H}_{2} \mathrm{O}$ to investigate the transport of groundwater sources [80]. Climatologists and paleontologists use stable isotopes of $\mathrm{O}_{2}$ to determine the past temperature of the Earth's atmosphere from ice cores [81. These examples represent just a few of the useful applications of stable isotope ratio measurements.

\subsection{Breath analysis}

One emerging application of trace gas detection is human breath analysis. Human breath contains trace quantities of more than 1000 molecules 82 . Abnormal concentrations for many of these different molecules, called 'biomarkers', have already been correlated to specific diseases and health conditions [83]. CE-DFCS is particularly well suited for breath analysis because of its wide spectral coverage allowing for detection of many different biomarkers simultaneously. In the $1.5-1.7 \mu \mathrm{m}$ region, there are more than 10 biomarkers that exist in human breath in detectable quantities. Figs. 23 and 24 show two sets of measurements that detect a couple of these biomarkers in human breath samples. These measurements were performed with the $1.5 \mu \mathrm{m}$ system described in the previous section. Detailed descriptions of these measurements can be found in [29].

The first set of breath measurement, shown in Fig. 23 , records absorptions of three different isotopes of $\mathrm{CO}_{2}$. This measurement is of a medical interest because the ratio of ${ }^{13} \mathrm{C}^{16} \mathrm{O}_{2}$ to ${ }^{12} \mathrm{C}^{16} \mathrm{O}_{2}$ can be used to determine if a patient is infected with Heliobacter pylori, a common cause of ulcers. This breath test is conducted by having the patient ingest a ${ }^{13} \mathrm{C}$-labeled urea and then measuring the ratio of ${ }^{13} \mathrm{C}^{16} \mathrm{O}_{2}$ to ${ }^{12} \mathrm{C}^{16} \mathrm{O}_{2}$ on the patients breath 84. Increased levels of ${ }^{13} \mathrm{C}^{16} \mathrm{O}_{2}$ indicate the presence of $H$. pylori, a bacteria that helps to convert the ${ }^{13} \mathrm{C}$ labeled urea into ${ }^{13} \mathrm{CO}_{2}$ before being exhaled in the patients breath. In the spectral region shown in Fig. 23,

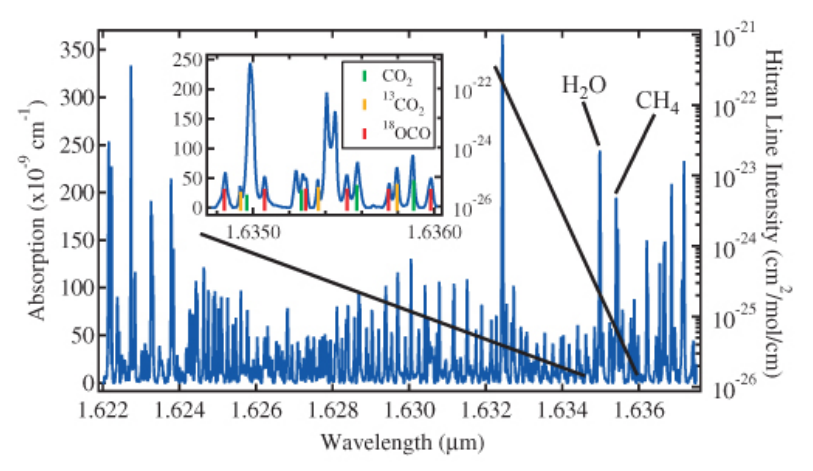

Fig. 23 a)Breath spectrum of $\mathrm{CO}_{2}$ isotopes near $1.63 \mu \mathrm{m}$. The inset shows a zoomed-in portion of the $\mathrm{CO}_{2}$ landscape. The spectral region between $1.622-1.638 \mu \mathrm{m}$ contains $78 \mathrm{CO}_{2}$ lines, $29{ }^{13} \mathrm{CO}_{2}$ lines, and $62{ }^{18} \mathrm{O}^{12} \mathrm{C}^{16} \mathrm{O}$ lines making it an ideal spectral region for determining isotope ratios. 
there are $78 \mathrm{CO}_{2}$ absorption lines and $29{ }^{13} \mathrm{CO}_{2}$ lines. The absorption due to each of these lines is used to determine the final ${ }^{13} \mathrm{C}^{16} \mathrm{O}_{2}$ to ${ }^{12} \mathrm{C}^{16} \mathrm{O}_{2}$ ratio which achieves an accuracy of $0.4 \%$.

The second breath measurement shown in Fig. 24 illustrates how environmental and behavioral factors that determine health can be monitored via breath analysis. Figure 24 shows the breath spectra of two students. Student 1 is a smoker who had a cigarette 15 minutes prior to providing the breath sample. Student 2 is a non-smoker who has relatively low exposure to carbon monoxide. Figure 24 shows that the breath of student 1 has a high $\mathrm{CO}$ concentration $(6 \mathrm{ppm})$, student 2 has a

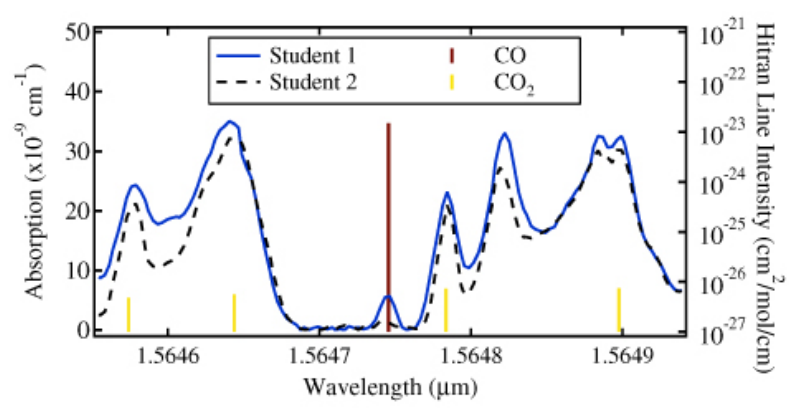

Fig. 24 a)Breath spectra near $1.565 \mu \mathrm{m}$ showing the difference between $\mathrm{CO}$ concentration in the breath of student 1 (smoker) versus student 2 (non-smoker). The vertical bars indicate the wavelengths and line intensities of $\mathrm{CO}_{2}$ and $\mathrm{CO}$ lines according to the HITRAN database.

low CO concentration $(<1 \mathrm{ppm})$. While smoking provides an example of the behaviorial effect on the $\mathrm{CO}$ level in breath, environmental conditions can also lead to elevated $\mathrm{CO}$ breath levels. For instance, individuals who spend a large portion of their day in congested traf- fic or people live with elevated levels of $\mathrm{CO}$ at home are also prone to elevated $\mathrm{CO}$ levels in their breath 85 .

The breath measurements presented here provide a modest glimpse of the health information that can be acquired by analyzing a patients breath. In the $1.5 \mu \mathrm{m}$ spectral region alone there are also strong absorptions from ammonia which can be used to detect kidney failure [86], acetone which is an indicator for diabetes [87, and ethane and ethylene for detecting lung cancer [2].

\subsection{Spectroscopy of cold molecules}

So far, measurements presented in this work have been on room temperature gas samples at relatively high pressures and therefore it is not required to employ higher spectral resolution than what is provided by the VIPA spectrometer. In this Section, we discuss a technique that uses precise control of the comb and cavity frequencies in conjunction with a VIPA spectrometer to extend the spectral resolution far beyond what is provided by the VIPA alone. We present some measurements on a cold molecular beam of $10 \% \mathrm{C}_{2} \mathrm{H}_{2}$ in a supersonic expansion of argon carrier gas that demonstrate these extended capabilities.

The first step to performing these measurements is to stabilize either the cavity or comb modes. In the measurements presented here, the cavity modes are stabilized as shown in Fig. 25. In this system, the frequency comb $f_{r}=99 \mathrm{MHz}$ and the cavity $F S R=396 \mathrm{MHz}$. This mismatch of $f_{r}$ and FSR has the unfortunate side 


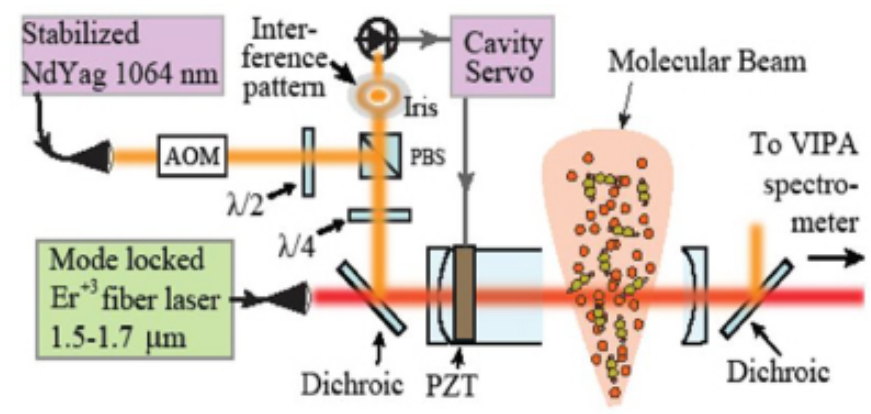

Fig. 25 Schematic of the cold molecular beam detection system. An iodine stabilized Nd:YAG laser stabilizes the cavity mode frequencies to $\Delta \nu=250 \mathrm{kHz}$. An $\mathrm{Er}^{+3}$ fiber comb is coupled into a $\mathcal{F}=6380$ and $\mathrm{FSR}=400 \mathrm{MHz}$ cavity that is oriented perpendicular to a pulsed supersonic beam. The cavity transmitted beam is dispersed and detected using a VIPA spectrometer.

effect that only $25 \%$ of the comb components are coupled into the cavity for spectroscopy. Ideally, a laser with a high $f_{r}\left(400 \mathrm{MHz} \leq f_{r} \leq 800 \mathrm{MHz}\right)$ would be coupled to a cavity of the equivalent $F S R$. Since a high- $f_{r}$ laser was not available, we used a high- $F S R$ cavity to make the frequency spacing between the comb modes in cavity transmission close to the resolution of the VIPA spectrometer. This configuration allows high resolution features to be retrieved with high measurement contrast.

The diagram in Fig. 26 provides a visual description of how higher resolution is achieved even if the cavity $F S R$ is not greater than the VIPA resolution. When one of the cavity modes within the resolution envelope of the VIPA experiences loss due to absorption, the VIPA channel registers a loss that is diluted by the number of

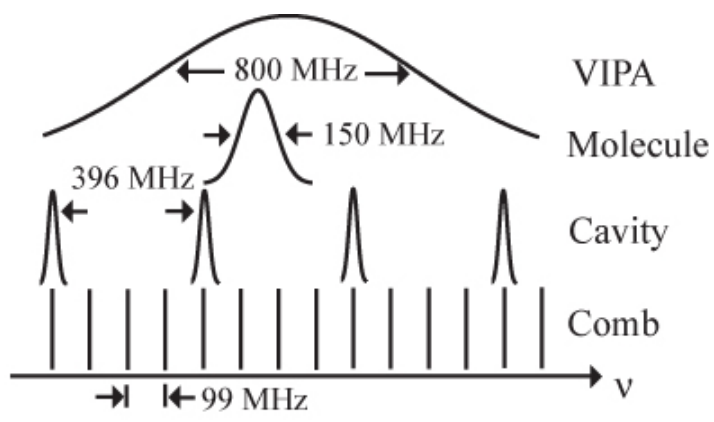

Fig. 26 High resolution can be achieved using a combination of VIPA resolution and frequency stabilized cavity modes.

non-interacting modes contained within the VIPA resolution envelope. If only a single mode lies within each VIPA pixel, then the absorption experienced by this single mode can be extracted, leading to an enhanced signal contrast and improved spectral resolution. In the case of measurements presented here, $\sim 4$ comb modes are present under each VIPA resolution envelope. The effect of multiple comb modes under each VIPA resolution channel is to dilute the absorption signal recorded by that channel. The relatively large number of comb modes per VIPA resolution channel is due to the Lorentzian transfer function of the VIPA. By stabilizing the comb with respect to the cavity, precise frequency sweep of the comb and cavity modes can then be executed to ensure that only one comb mode is coupled out at a time under the VIPA resolution. A high-resolution absorption landscape can then be mapped out with the resolution limited only by the linewidth of a single comb component.

For the cold $\mathrm{C}_{2} \mathrm{H}_{2}$ measurements presented here, the cavity was stabilized using an iodine-stabilized Nd:YAG 
laser at $1.064 \mu \mathrm{m}$. The cavity length was locked to within $\lambda / 3200$, resulting in a frequency stability of each cavity mode of $250 \mathrm{kHz}$. The cavity modes were then scanned using an acousto-optic modulator (AOM) that varied the frequency of the Nd:YAG laser. To scan a complete $F S R$ at $1.5 \mu \mathrm{m}$, the frequency of the $1.064 \mu$ laser must be scanned 1.5/1.064 × $400 \mathrm{MHz} \approx 600 \mathrm{MHz}$. Figure 27 shows a full FSR scan of the cavity modes at the peak absorption wavelengths of eight of the lowest lying rotational lines in the $\nu_{1}+\nu_{3} \mathrm{C}_{2} \mathrm{H}_{2}$ overtone spectrum. Due to the broadband nature of the VIPA spectrometer, these traces (plus thousands of others) were recorded during a single frequency scan by the AOM.

The information recovered from scans such as those shown in Fig. 27 is substantial. Due to the high frequency resolution of this technique, accurate lineshapes of the molecular absorption can be measured to extract both velocity and collision rate information for the beam. The broadband nature of this method allows for simultaneous acquisition of absorption information for many rovibrational lines, yielding rapid temperature measurements for the internal degrees of freedom of the molecule. Finally, because the absorption loss is normalized to the mirror loss, the density of the molecular beam can be characterized to high precision. Combining these capabilities while making measurements across different beam positions allows complete characterization of a cold molecular beam.

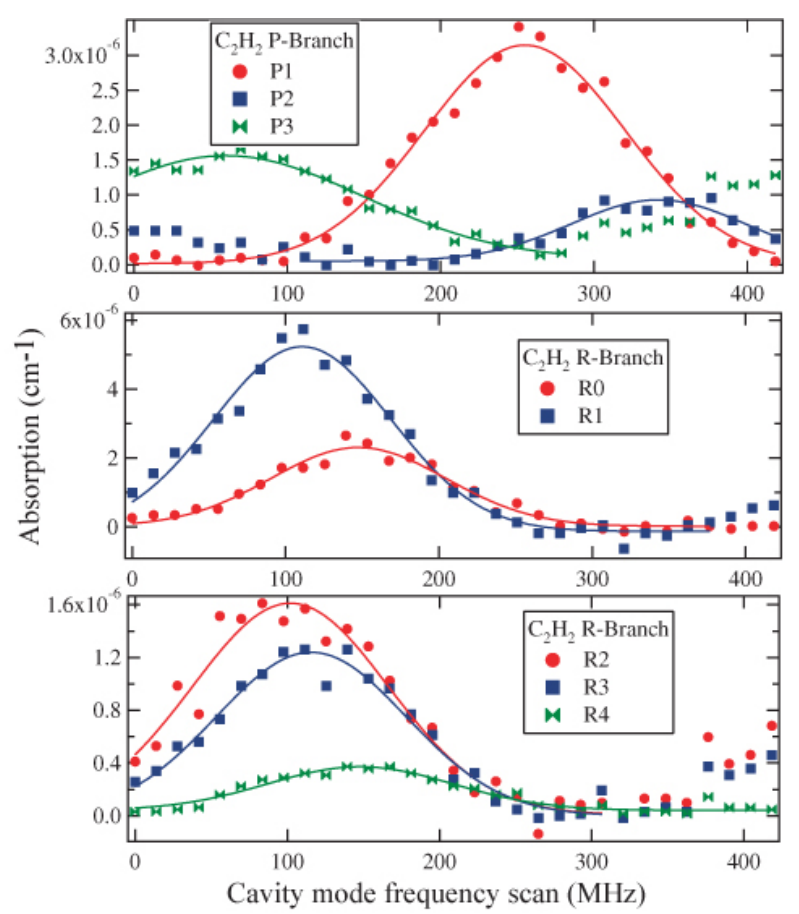

Fig. 27 Precise scans of the cavity mode frequencies over one cavity FSR (400 MHz), revealing spectroscopic features with linewidths below the VIPA and the FSR resolution limits. Panels (a), (b), and (c) display the lowest R- and P-branch rotational lines of the $\mathrm{C}_{2} \mathrm{H}_{2} \nu_{1}+\nu_{3}$ overtone band detected in a supersonic cold molecular beam, yielding a complete characterization of the velocity, internal energy, and density distributions of the beam.

\section{Conclusion}

The measurements and techniques presented in this review represent the current state of the art for CE-DFCS. As this is a relatively new field, the capabilities of these systems and the techniques available are improving rapidly. Already CE-DFCS has demonstrated detection of molecular concentrations in the low ppb range for many molecules with spectral resolutions as low as $250 \mathrm{kHz}$. The remarkable aspect about CE-DFCS is that this high sensitivity 
and resolution can be implemented across large spectral bandwidths, realizing a true real-time scenario for rapid detection.

As applications of these systems become more demanding, the current limits of CE-DFCS systems will certainly be overcome. Directions for improving CE-DFCS performance include new broadband cavity designs such as prism cavities mentioned in Section 3.3. Such cavities would greatly increase the number of molecules that can be probed with a single CE-DFCS system. Also, extending precise frequency comb sources to the mid-IR spectral region, with a reasonable useful power, will allow much enhanced absorption sensitivity by detection of strong fundamental vibration transitions, reducing the minimum detectable concentration of many molecules to the parts per trillion level.

Aside from trace detection, new applications for CE- sis via CE-DFCS. F. Adler and K. Cossel are helping further expand the capabilities and various applications of CE-DFCS. We also thank T. Schibli, A. Pe'er, D. Yost, S. Diddams, and D. Hudson for many useful discussions and technical help. Funding support is provided by AFOSR, DARPA, NIST, and NSF.

\section{References}

1. S. Y. Lehman, K. A. Bertness, J. T. Hodges: J. Cryst. Growth 250, 262 (2003).

2. C. S. Patterson, L. C. McMillan, C. Longbottom, G. M. Gibson, M. J. Padgett, K. D. Skelton: Meas. Sci. Technol. 18, 1495 (2007).

3. S. Spuler, M. Linne, A. Sappey, S. Snyder: Appl. Opt. 39, $2480(2000)$.

4. M. W. Todd, R. A. Provencal, T. G. Owano, B. A. Paldus, A. Kachanov, K. L. Vodoyanov, M. Hunter, S. L. Coy, J. I. Steinfeld, J. T. Arnold: Appl. Phys. B 75, 367 (2002). DFCS include detection and characterization of cold moleculȩ, J. Ye, L.-S. Ma, and J. L. Hall, J. Opt. Soc. Am. B 15, 6 including their dynamics and interactions. High-resolution (1998)

coherent control of atomic and molecular systems similar to work already performed using free-space DFCS [19] 20] represents another exciting possibility.

We thank many of our colleagues who have contributed to the work presented here. In particular, A. Marian and M. Stowe were involved in the initial demonstration of direct frequency comb spectroscopy. R. J. Jones and K. Moll helped develop the cavity-enhanced frequency comb spectroscopy. D. Balslev-Clausen and M. Kirchner participated in the demonstration of breath analy-

6. E. H. Wahl, S. M. Tan, S. Koulikov, B. Kharlamov, C. R. Rella, and E. R. Crosson, Optics Express. 14, 1673 (2006). 7. A. A. Kosterev, A. L. Malinovsky, F. K. Tittel, C. Gmachl, F. Capasso, D. L. Sivco, J. N. Baollargeon, A. L. Hutchinson, A. Y. Cho: Appl. Opt. 40, 5522 (2001).

8. M.W. Todd, R.A. Provencal, T.G. Owano, B.A. Paldus, A. Kachanov, K.L. Vodopyanov, M. Hunter, S.L. Coy, J.I. Steinfeld and J.T. Arnold: Appl. Phys. B. 75, 367 (2002). 9. K. W. Jucks, D. G. Johnson, K. V. Chance, W. A. Traub, J. J. Margitan, G. B. Osterman, R. J. Salawitch, and Y. Sasano: Geophys. Res. Lett. textbf25, 3935 (1998). 
10. J. N. Begum: J. Quant. Spectrosc. Radiat. Transfer. 95, 423 (2005).

11. K. K. Lehmann, B. H. Pate, G. Scoles: Laser Chem. 11, 237 (1991)

12. J. E. Gambogi, E. R. Th. Kerstel, K. K. Lehmann, and G. Scoles: J. Chem. Phys. 100, 2612 (1994).

13. H.R. Thorsheim, J.Weiner, P.S. Julienne, Phys. Rev. Lett. 58, 2420 (1987).

14. E. R. Hudson, C. Ticknor, B. C. Sawyer, C. A. Taatjes, H. J. Lewandowski, J. R. Bochinski, J. L. Bohn, and J. Ye, Phys. Rev. A 73, 063404 (2006).

15. J. Doyle et al., Eur. Phys. J. D 31, 149 (2004).

16. D. Wang et al.: Eur. Phys. J. D 31, 165 (2004).

17. S. Ospelkaus, A. Pe'er, K.-K. Ni, J. J. Zirbel, B. Neyenhuis, S. Kotochigova, P. S. Julienne, J. Ye, and D. S. Jin, Nature Physics, submitted (2008).

18. A. Marian, M. C. Stowe, J. R. Lawall, D. Felinto, J. Ye: Science. 306, 2063 (2004).

19. M. C. Stowe, F. C. Cruz, A. Marian, J. Ye: Phys. Rev. Lett. 96, 153001-1-4 (2006).

20. M. C. Stowe, M. J. Thorpe, A. Pe'er, J. Ye, J. E. Stalnaker, V. Gerginov and S. A. Diddams: AAMOP 55, 1 (2008).

21. L. E. Nelson, D. J. Jones, K. Tamura, H. A. Haus, E. P. Ippen: Appl. Phys. B 65, 277 (1997).

22. F. Tauser, F. Adler, A. Leitenstorfer: Opt. Lett. 29, 516 (2004)

23. J. K. Ranka, R. S. Windeler, and A. J. Stentz: Opt. Lett. $\mathbf{2 5}, 25(2000)$.

24. F. Keilmann, C. Gohle, R. Holzwarth: Opt. Lett. 29, $1542(2004)$
25. S. A. Diddams, L. Hollberg, V. Mbele: Nature 445, 627 (2007).

26. T. Gherman, D. Romanini: Opt. Express 10, 1033 (2002).

27. M. J. Thorpe, K. D. Moll, B. Safdi, J. Ye: Science 311, 1595 (2006).

28. M. J. Thorpe, D. D. Hudson, K. D. Moll, J. Lasri, J. Ye: Opt. Lett. 32, 307 (2007).

29. C. Gohle, B. Stein, A. Schliesser, T. Udem, T. W. Hänsch: Phys. Rev. Lett. 99, 263902 (2007).

30. M. J. Thorpe, D. Balslev-Clausen, M. Kirchner, J. Ye: Opt. Express. 16, 2387 (2008).

31. S. T. Cundiff, J. Ye, J. L. Hall: Rev. Sci. Instrum. 72, 3749 (2001).

32. J. Ye, S. T. Cundiff: Femtosecond Optical Frequency Comb Technology: Principle, Operation, and Application. (Springer, New York 2005) 16-28.

33. R. J. Jones, I. Thomann, J. Ye: Phys. Rev. A. 69, 051803 (2004).

34. T. A. Birks, W. J. Wadsworth, and P. St. J. Russell: Opt. Lett. 25, 1415 (2000).

35. D. J. Jones, S. A. Diddams, J. K. Ranka, A. Stentz, R. S. Windeler, J. L. Hall, S. T. Cundiff: Science. 288, 635 (2000).

36. T. M. Fortier, D. J. Jones, S. T. Cundiff: Opt. Lett. 28, 2198 (2003).

37. R. Ell, U. Morgner, F. X. Kärtner, J. G. Fujimoto, E. P. Ippen, V. Scheuer, G. Angelow, T. Tschudi, M. J. Lederer, A. Boiko, B. Luther-Davies: Opt. Lett. 26, 373, (2001). 38. T. M. Fortier, A. Bartels, and S. A. Diddams: Opt. Lett. 31, 1011, (2006). 
39. T. Gherman, S. Kassi, A. Campargue, D. Romanini: Chem. Phys. Lett. 383, 353 (2004).

40. S. M. Foreman, D. J. Jones, J. Ye: Opt. Lett. 28, 370 (2003).

41. E. Penman, C. McGowan, P. Loza-Alvarez, D. T. Reid, M. Ebrahimzadeh, W. Sibbett: J. Mod. Opt. 45, 1285 (1998)

42. D. Mazzotti, P. Cancio, G. Giusfredi, P. De Natale, M. Prevedelli: Opt. Lett., Vol. 30, 997 (2005).

43. A. Schliesser, M. Brehm, F. Keilmann, D. W. van der Weide: Opt. Express 13, 9029 (2005).

44. R. J. Jones, K. D. Moll, M. J. Thorpe, J. Ye: Phys. Rev. Lett. 94, 193201-1-4 (2005).

45. C. Gohle, T. Udem, M. Herrmann, J. Rauschenberger, R. Holzwarth, A. Schuessler, F. Krausz, T. W. Hänsch: Nature 436, 234 (2005).

46. D. C. Yost, T. R. Schibli, and J. Ye, Opt. Lett., submitted (2008))

47. E. E. Eyler, D. E. Chieda, M. C. Stowe, M. J. Thorpe, T. R. Schibli, and J. Ye, Eur. Phys. J. D, DOI: 10.1140/epjd/e2007-00289-y (2007).

48. F. Tauser, A. Leitenstorfer, W. Zinth: Opt. Express 11, 594-600 (2003),

49. B. R. Washburn, S. A. Diddams, N. R. Newbury, J. W. Nicholson, M. F. Yan, C. G. Jrgensen: Opt. Lett. 29, 250$252(2004)$

50. P. Pal, W. H. Knox, I. Hartl, M. E. Fermann: Opt. Express textbf15, 12161 (2007).

51. T. R. Schibli, D. C. Yost, M. J. Martin, J. Ye, I. Hartl, A. Marcinkevicius, and M. E. Fermann, Nature Photonics, in press (2008).
52. P. Balling, M. Fischer, P. Kubina, R. Holzwarth: Opt. Express 13, 9196 (2005).

53. K. Moutzouris, F. Adler, F. Sotier, D. Trutlein, A. Leitenstorfer: Opt. Lett. 31, 1148 (2006).

54. C. Erny, K. Moutzouris, J. Biegert, D. Khlke, F. Adler, A. Leitenstorfer: Opt. Lett. 32, 1138 (2007).

55. K. K. Lehmann, P. Rabinowitz: U. S. Patent 5973864 (1999).

56. E. Hecht: Optics 3rd ed. (Addison-Wesley, New York 1998) 409-417.

57. A. O'Keefe, D. A. G. Deacon: Rev. Sci. Instrum. 59, 2544 (1988).

58. R. G. DeVoe, C. Fabre, K. Jungmann, J. Hoffnagle, R. G. Brewer: Phys. Rev. A. 371802 (1988).

59. C. J. Hood, H. J. Kimble, J. Ye: Phys. Rev. A. 64, 033804 (2002).

60. M. J. Thorpe, R. J. Jones, K. D. Moll, J. Ye, R. Lalezari: Opt. Express 13, 882 (2005).

61. The work at JILA has greatly benefited from collborative coating runs from R. Lalezari of Advanced Thin Films. Mention of company or product name is for the purpose of technical communications and does not represent an endorsement of NIST.

62. R. J. Jones and J. C. Diels: Phys. Rev. Lett. 86, 3288 (2001).

63. J. Ye, T. W. Lynn: Adv. At. Mol. Opt. Phys. 49, 1 (2003).

64. L. Gianfrani, R. W. Fox, L. Hollberg: J. Opt. Soc. Am. B 16, 2247 (1999).

65. J. Ye, L. S. Ma, and J. L. Hall: Opt. Lett. 21, 1000 (1996). 
66. L. S. Ma, J. Ye, P. Dube, and J. L. Hall: J. Opt. Soc. Am. B. 16, 2255 (1999).

67. J. Poirson, F. Bretenaker, M. Vallet, A. Le Floch: J. Opt. Soc. Am. B 14, 2811 (1997).

68. R. Bakowski, L. Corner, G. Hancock, R. Kotchie, R. Peverall, G. A. D. Ritchie: Appl. Phys. B 75, 745 (2002).

69. D. Romanini, K. K. Lehmann: J. Chem. Phys. 99, 6287 (1993).

70. M. D. Levenson, B. A. Paldus, T. G. Spence, C. C. Harb, J. S. Harris, Jr., and R. N. Zare: Chem. Phys. Lett. 290, 335 (1998).

71. J. J. Scherer, J. B. Paul, A. OKeefe, and R. J. Saykally: Chem. Rev. 97, 25 (1997).

72. J. Ye, J. L. Hall: Phys. Rev. A 61, 061802 (2000).

73. S. Xiao,A. M. Weiner: Opt. Express 12, 2895 (2004).

74. A. Vega, A. M. Weiner, C. Lin: Appl. Opt. 42, 4152 (2003).

75. J. J. Scherer et al.: Appl. Opt. 40, 6725 (2001).

76. G. P. Agrawal: Nonlinear Fiber Optics 4th ed. (Academic Press, New York 2007) 162-168.

77. L. S. Rothman et al: J. Quant. Spectrosc. Radiat. Transfer. 96, 139 (2005).

78. L. Lundsberg-Nielsen, F. Hegelund, F. M. Nicolaisen: J. Mol. Spectrosc. 162, 230 (1993).

79. E. R. Crosson et al.: Anal. Chem. 74, 2003 (2002).

80. L. N. Plummer, E. Busenberg, J. K. Bohlke, D. L. Nelms,

R. L. Michel, P. Schlosser: Chem. Geol. 179, 93 (2001).

81. E. Mosley-Thompson, L. G. Thompson, P.N. Lin: Ann. Glaciology 43, 42 (2006).

82. T. H. Risby, S. F. Solga: Appl. Phys. B. 85, 421 (2006).

83. W. Cao, Y. Duan: Clin. Chem. 52, 800 (2006).

84. P. D. Klein, D. Y. Graham: Am. J. Gastroenterol. 88, 1865 (1993).

85. A. J. Cunnington, P. Hormbrey: Postgraduate Med. J. 78, 233 (2002).

86. J. Manne, O. Sukhorukov, W. Jager, J. Tulip: Appl. Opt. 45, 9230 (2006).

87. C. Wang, S. T. Scherrer, D. Hossain: Appl. Opt. 58, 784 (2004). 